\title{
Pathomechanisms of ALS8: altered autophagy and defective RNA binding protein (RBP) homeostasis due to the VAPB P56S mutation
}

Priyanka Tripathi ${ }^{1,2}$, Haihong Guo ${ }^{1}$, Alice Dreser ${ }^{1}$, Alfred Yamoah ${ }^{1,2}$, Antonio Sechi ${ }^{3}$, Christopher Marvin Jesse $\mathbb{B}^{1,4}$,

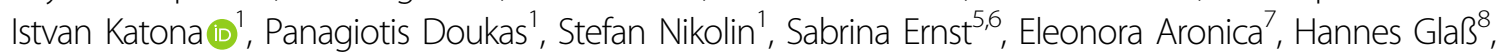
Andreas Hermann ${ }^{8,9}$, Harry Steinbusch ${ }^{2,10}$, Alfred C. Feller ${ }^{11}$, Markus Bergmann ${ }^{12}$, Dick Jaarsma ${ }^{13}$, Joachim Weis ${ }^{1}$ and Anand Goswami (10)

\begin{abstract}
Mutations in RNA binding proteins (RBPs) and in genes regulating autophagy are frequent causes of familial amyotrophic lateral sclerosis (fALS). The P56S mutation in vesicle-associated membrane protein-associated protein B (VAPB) leads to fALS (ALS8) and spinal muscular atrophy (SMA). While VAPB is primarily involved in the unfolded protein response (UPR), vesicular trafficking and in initial steps of the autophagy pathway, the effect of mutant P56SVAPB on autophagy regulation in connection with RBP homeostasis has not been explored yet. Examining the muscle biopsy of our index ALS8 patient of European origin revealed globular accumulations of VAPB aggregates co-localised with autophagy markers LC3 and p62 in partially atrophic and atrophic muscle fibres. In line with this skin fibroblasts obtained from the same patient showed accumulation of P56S-VAPB aggregates together with LC3 and p62. Detailed investigations of autophagic flux in cell culture models revealed that P56S-VAPB alters both initial and late steps of the autophagy pathway. Accordingly, electron microscopy complemented with live cell imaging highlighted the impaired fusion of accumulated autophagosomes with lysosomes in cells expressing P56S-VAPB. Consistent with these observations, neuropathological studies of brain and spinal cord of P56S-VAPB transgenic mice revealed signs of neurodegeneration associated with altered protein quality control and defective autophagy. Autophagy and RBP homeostasis are interdependent, as demonstrated by the cytoplasmic mis-localisation of several RBPs including pTDP43, FUS, Matrin 3 which often sequestered with P56S-VAPB aggregates both in cell culture and in the muscle biopsy of the ALS8 patient. Further confirming the notion that aggregation of the RBPs proceeds through the stress granule (SG) pathway, we found persistent G3BP- and TIAR1-positive SGs in P56S-VAPB expressing cells as well as in the ALS8 patient muscle biopsy. We conclude that P56S-VAPB-ALS8 involves a cohesive pathomechanism of aberrant RBP homeostasis together with dysfunctional autophagy.
\end{abstract}

\footnotetext{
Correspondence: Anand Goswami (agoswami@ukaachen.de)

${ }^{1}$ Institute of Neuropathology, RWTH Aachen University Medical School,

Pauwelsstr. 30, 52074 Aachen, Germany

2EURON - European Graduate School of Neuroscience, Maastricht, The

Netherlands

Full list of author information is available at the end of the article

These authors contributed equally: Priyanka Tripathi, Haihong Guo, Joachim

Weis, Anand Goswami

Edited by M. Agostini
}

\section{Introduction}

Amyotrophic lateral sclerosis (ALS) is a devastating disease characterised by progressive loss of upper and lower motor neurons (MNs), eventually leading to paralysis and death. Majority of the fALS- linked genes either regulate protein quality control and autophagy and/ or RNA binding protein (RBP) homeostasis ${ }^{1,2}$. Pathogenic mutations in these genes induce neuronal toxicity coupled

\section{(c) The Author(s) 2021}

(c) (i) Open Access This article is licensed under a Creative Commons Attribution 4.0 International License, which permits use, sharing, adaptation, distribution and reproduction cc) in any medium or format, as long as you give appropriate credit to the original author(s) and the source, provide a link to the Creative Commons license, and indicate if changes were made. The images or other third party material in this article are included in the article's Creative Commons license, unless indicated otherwise in a credit line to the material. If material is not included in the article's Creative Commons license and your intended use is not permitted by statutory regulation or exceeds the permitted use, you will need to obtain permission directly from the copyright holder. To view a copy of this license, visit http://creativecommons.org/licenses/by/4.0/. 
with protein aggregation, defective proteostasis/autophagy and aberrant RBP homeostasis. However, the factors that initially triggers these key events and the functional interplay between autophagy and RBPs homeostasis and their downstream signalling are poorly understood thus far.

A dominantly inherited mutation (P56S) in the gene encoding for the vesicle-associated membrane proteinassociated protein $B$ (VAPB) has been associated with typical ALS (ALS8), atypical ALS and late-onset spinal muscular atrophy (SMA) ${ }^{3-5}$. VAPB is a ubiquitously expressed membrane-anchored protein of ER and ERGolgi intermediate vesicles ${ }^{6,7}$. VAPB contains an $\mathrm{N}$ terminal major sperm protein (MSP), a coiled coil and a transmembrane domain. The MSP domain binds to a variety of proteins from various cellular compartments that contain FFAT motifs (two phenylalanines in an acidic region) and that are known to orchestrate multiple functions including retrograde transport of proteins ${ }^{6}$, lipid transfer toward the $\mathrm{Golgi}^{8}$, regulation of ER structure through interaction with the microtubule network ${ }^{9,10}$ and modulation of responses to ER stress ${ }^{11-13}$. In line with this, recent studies have shown that VAPB is involved in ER-orchestrated autophagosome biogenesis ${ }^{14}$.

P56S-VAPB forms detergent insoluble cytoplasmic inclusions, which exhibits toxic gain of functions as shown by others ${ }^{12,13,15-18}$ including the detrimental effects of aggregated P56S-VAPB on protein quality control, ER-organelle tethering, mitochondrial toxicity, UPR imbalance and alterations in endo-lysosomal pathways ${ }^{11,13,19-21}$. P56S-VAPB transgenic $(\mathrm{Tg})$ mice under the control of a pan-neuronal promoter Thy1.2 showed signs of ER stress in both spinal MNs as well as in CNS neurons accompanied by a minor degeneration of CNS neurons ${ }^{22}$. Lumbar spinal cord $\alpha$-MNs of these mice can develop cytoplasmic TDP-43 accumulation ${ }^{23}$, but showed only incomplete degeneration accompanied by a mild motor phenotype at 18 months of age ${ }^{23}$. In line with this observation, P56S-VAPB knock-in mice displayed induction of ER stress and autophagic responses in $\alpha-\mathrm{MNs}$ before obvious onset of behavioural defects ${ }^{24}$. In addition to the toxic gain of function P56S-VAPB has been shown to induce a dominant negative mechanism by incorporating the normal wild-type (wt) VAPB, supporting a loss of function hypothesis for ALS8 pathogenesis ${ }^{13,16,17}$. Consistent with these data, VAPB knockdown in zebrafish led to swimming deficits and VAPB knockout mice showed mild motor deficits associated with autophagy defects $^{25}$. Taken together, these results suggest that VAPB is involved in many functions which are crucial for neuronal survival and that P56S-VAPB alters these processes involving both toxic gain and/or loss of function. However, it is still not clear how these pathways are interconnected and pathologically orchestrated in ALS8 to induce neurodegeneration and if and how P56S-VAPB modulates these converging mechanisms.

To address these issues, we focussed on the effect of P56S-VAPB on autophagy and RBP homeostasis. We found that P56S-VAPB-mediated ALS pathology is closely linked to alterations of both of these interdependent cellular processes. Motor neurons are known to be particularly vulnerable to altered autophagy and RBP homeostasis ${ }^{1,26-30}$, explaining why they are selectively affected by a mutation that is altering these pathways simultaneously (see also schematic representation, Scheme 1)

\section{Materials and methods \\ Reagents and antibodies}

Fluorescent nucleic acid stain Hoechst 33258 was purchased from Molecular Probes. Thapsigargin, MG132, Tunicamycin, EGF, Rapamycin, Bafilomycin A, HEPES 4(2-hydroxyethyl)-1-piperazineethanesulfonic acid were purchased from Sigma Aldrich. All primary and secondary antibodies and their dilutions used in this study are listed in Supplementary Table 1. Many of these antibodies have been used by us in previous published studies (see references in Supplementary Table 1). Rabbit polyclonal VAPB antibody was custom made and used by us in previous studies ${ }^{19,31-33}$

\section{Human muscle biopsies}

Autopsy tissue from P56S-VAPB is not available so far. We could use archival, formalin-fixed, paraffin-embedded muscle biopsy materials obtained from an index patient from European origin with ALS8 due to the P56S-VAPB mutation $^{5}$ and stain cryostat sections and resin blocks of glutaraldehyde-fixed muscle tissue from his mother, who had shown similar symptoms as her son, but had died before genetic diagnosis had been available. Muscle biopsies from clinically confirmed sporadic ALS as well as age-matched control muscle biopsies were also obtained from the archives of the Institute of Neuropathology, RWTH Aachen University Hospital, following the guidelines of the Ethics Committee of RWTH Aachen University Hospital (also see below ethical statement).

\section{Experimental animals (P56S-VAPB transgenic mice)}

VAPB transgenic mice had been generated using the cDNAs of wild-type or P56S-mutant human VAPB cloned into the Thy1.2-expression module. The VAPB constructs also contained an HA-tag to enable easy visualisation of transgenic VAPB by immunocytochemical and biochemical approaches. Details of the generation of this transgenic mouse are described elsewhere ${ }^{15}$. In this study we used tissue from Thy1 wt-VAPB-HA and Thy1 P56SVAPB-HA mice at the age around 200 days. Animals were not randomly assigned during collection, but the strain, 


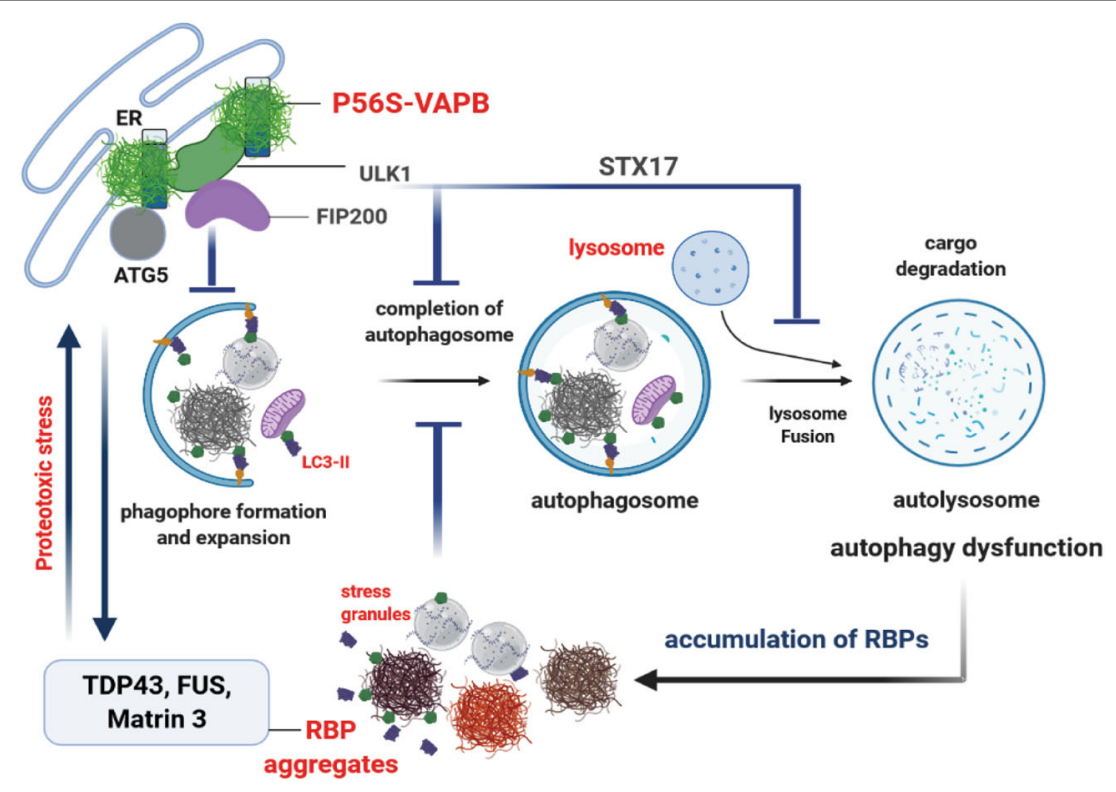

Scheme 1 Proposed cohesive pathomechanism of aberrant RBPs hoemostasis and autophagy in ALS8. P56S-VAPB aggregates alter early autophagy proteins ULK1, ATG5-ATG12; late autophagy protein STX17 and leads to the defects in both early and late steps of autophagy. P56S-VAPB mediated proteotoxic stress together with altered autophagy might contribute to dysregulations of several fALS-associated RBPs (TDP-43, FUS, Matrin 3) including the SGs and can initiate a vicious cycle. (Schematic image was generated with the BioRender software).

sex, and age of the mice were the same. We used paraffin sections of formalin-fixed material from these animals for most of the histological analyses and fresh frozen tissue for Western blot analyses. Immunohistochemistry protocols for formalin-fixed, paraffin-embedded mouse tissue and all the antibodies used (Supplementary Table 1) were optimised and performed in analogy to the protocol used for human biopsy and autopsy tissue to avoid any discrepancies. All procedures including housing and handling the animals were done in accordance with the "Principles of laboratory animal care" (NIH publication No. 86-23) and the guidelines of the Erasmus University (The Netherlands) animal care committee.

\section{Cell culture, transient transfection and treatments \\ Cell culture and treatment}

Human epithelial cancer cells (HeLa), Human embryonic kidney cell line (HEK-293), African green monkey kidney cells (COS-7) and NSC34 motor neuronlike cells were cultured in Dulbecco's modified Eagle's medium (DMEM, Invitrogen, Carlsbad, CA, USA), supplemented with $10 \% \mathrm{FBS}$ and $1 \%$ antibiotic/anti-mycotic solution (Invitrogen). Mouse embryonic fibroblasts (MEF) obtained from the GFP-LC3 transgenic mice ${ }^{34}$ were also maintained in DMEM supplemented with $10 \% \mathrm{FBS}$ and $1 \%$ antibiotic/anti-mycotic solution. The human epidermoid carcinoma cell line A431 was grown in Dulbecco's modified Eagle's medium (DMEM, Invitrogen, Carlsbad, CA, USA) supplemented with $10 \%$ FBS and
$0.1 \%$ Gentamycin. NIH-3T3 cells stably expressing GFPLC3 or tandem mCherry-EGFP-LC3 were cultured in DMEM supplemented with 10\% FBS, 1\% penicillin/ streptomycin and puromycin (Sigma Aldrich). Fibroblasts obtained from P56S-ALS8 fALS patients and healthy controls were cultured in Dulbecco's modified Eagle's medium (DMEM, Invitrogen, Carlsbad, CA, USA), supplemented with $10 \% \mathrm{FBS}$ and $1 \%$ antibiotic/anti-mycotic solution (Invitrogen) 20\% FBS, 1\% penicillin/streptomycin, $1 \%$ L-Glutamin and $0.056 \%$ amphotericin. Cells were maintained in a humidified incubator at $37^{\circ} \mathrm{C}$ and $5 \%$ $\mathrm{CO}_{2}$. Generation of NIH-3T3 cells stably expressing GFPLC3 or tandem mCherry-EGFP-LC3 with retroviral infection is described elsewhere ${ }^{35}$.

\section{Human IPSc-derived motor neurons (MNs)}

We used iPSCs generated from fibroblasts of a healthy individual donor (male, age at skin biopsy 34). The generation and characterisation of the line was previously described $^{36}$. For MNs differentiation, we first derived neural precursor cells (NPCs) which were maintained and differentiation into MNs as shown previously ${ }^{37}$. In brief, NPC were maintained in basic medium (DMEM-F12/ Neurobasal 50:50 medium, N2 Supplement (1:200), B27 Supplement without vitamin A (1:100), penicillin/streptomycin (1\%), GlutaMAX (1\%)), supplemented with Chiron $99021(3 \mu \mathrm{M})$, Ascorbic acid $(150 \mu \mathrm{M})$ and Purmorphamine $(0.5 \mu \mathrm{M})$ on the dishes coated with Matrigel. For induction of the differentiation into motor neurons, 
NPC were split on the Matrigel coated dish in the basic medium supplemented with BDNF $(1 \mathrm{ng} / \mathrm{ml})$, Ascorbic acid $(200 \mu \mathrm{M})$, Retinoic acid $(1 \mu \mathrm{M})$, GDNF $(1 \mathrm{ng} / \mathrm{ml})$ and Purmorphamine $(0.5 \mu \mathrm{M})$ and maintained for 5 days. For the final maturation, the medium was changed on the day 6 to the basic medium supplemented with DBcAMP $(100 \mu \mathrm{M})$, BDNF $(2 \mathrm{ng} / \mathrm{ml})$, Ascorbic acid $(200 \mu \mathrm{M})$, TGF $\beta-3(1 \mathrm{ng} / \mathrm{ml})$ and GDNF $(2 \mathrm{ng} / \mathrm{ml})$. Between day 7 and 10 the cells were split onto the dishes coated with Poly-L-ornithine and laminin and maintained for at least 4-5 weeks before they were used for the final analysis. The cells were regularly tested for mycoplasma contamination.

\section{Transient transfections}

Cells were transfected to express either GFP-wt-VAPB or GFP-P56S-VAPB. The detailed description of generation of these plasmids is given elsewhere ${ }^{31}$. The empty pEGFP-N1 vector was used as a transfection control. All cell lines were transfected using Lipofectamine 2000 reagent (Invitrogen) according to the manufacturer's recommendations. After $4 \mathrm{~h}$ incubation at $37^{\circ} \mathrm{C}$ and $5 \% \mathrm{CO}_{2}$ the transfection reagent containing medium was replaced by fresh medium and analysis was performed $48 \mathrm{~h}$ later. IPS-derived MNs were transfected using NeuroMag reagent (OZ Biosciences) according to the manufacturer's protocol; the analysis was performed after $48 \mathrm{~h}$.

\section{Immunocytochemistry}

HeLa, HEK-293, COS-7, MEFs and NIH-3T3 cells were cultured on $\mu$-dishes (ibidi, $\mathrm{GmbH}$ ) and transiently transfected either with GFP-wt-VAPB or P56S-VAPB. After $48 \mathrm{~h}$ cells were fixed in 4\% PFA and processed for confocal microscopy. Permeabilization with $0.5 \%$ Triton X100 and blocking with $4 \%$ skimmed milk/normal goat serum was followed by primary antibody incubation overnight at $4{ }^{\circ} \mathrm{C}$. Secondary Alexa488- or Alexa594conjugated anti-mouse or anti-rabbit antibodies (Invitrogen) were used for visualisation. Nuclei were stained with Hoechst $33258(1 \mu \mathrm{g} / \mathrm{ml})$ or were mounted with DAPI containing fluorescent mounting media (DAKO) and visualised using a Zeiss LSM 700 confocal microscope (Zeiss, Oberkochen, Germany). Images were processed using the Zeiss LSM software and Adobe Photoshop CS5.

\section{Stress granule analysis}

FRAP experiments for stress granules analysis on RFPG3BP cell lines ${ }^{38,39}$ overexpressing wt and P56S-VAPB were performed on a Zeiss LSM710 confocal microscope (Zeiss, Oberkochen, Germany) equipped with a cell incubator at $37{ }^{\circ} \mathrm{C}$ and $5 \% \mathrm{CO}_{2}$. The images were acquired using a Plan-Apochromat $\times 63 / 1.40$ Oil DIC M27 objective and analysed with ZEN 2012 software. For bleaching
90\% power of the $561 \mathrm{~nm}$ laser (max. $20 \mathrm{~mW}$ ) was used with the following capture settings: $380 \mathrm{~ms}$ per frame and one image every $600 \mathrm{~ms}$ with 10 and 100 frames before and after bleaching. The bleaching area covered $20 \times 20$ pixels with a pixel size of $0.1 \mu \mathrm{m}$. For all experiments the bleached area and the duration of the laser impulse were kept constant. The extent of recovery of the fluorescent signal was determined using ImageJ to measure the average pixel intensity values within three distinct regions of interest: ROI1: bleached area, ROI2: unbleached area within the cell and ROI3: background. Normalised FRAP recovery curves and the mobile fraction were calculated using the programme easy FRAP ${ }^{40}$.

\section{Live cell imaging to analyse RFP-GFP-LC3 fusion}

To analyse the dynamics of the RFP-GFP-LC3 fusion protein, GFP and RFP channels were acquired every minute for up to $4 \mathrm{~h}$ using the imaging system described above, see also ${ }^{35}$. The extent of autophagosome maturation was determined by measuring the co-localisation of the GFP and RFP signals as expressed by the Pearson's coefficient using ZEN software.

\section{Immunoblot analysis}

Cells were washed twice with ice-cold PBS and scraped off the culture plate. After centrifugation at $6000 \mathrm{rpm}$ for $5 \mathrm{~min}$ and removal of the supernatant, cell pellets were resuspended in lysis buffer ( $50 \mathrm{mM}$ Tris-Cl, pH8.0, $150 \mathrm{mM}$ $\mathrm{NaCl}, 1 \%$ Triton X-100 in PBS, $0.5 \mathrm{mM}$ PMSF and complete protease inhibitor mixture, Roche Applied Sciences) and incubated on ice for $30 \mathrm{~min}$ followed by sonication with an amplitude of $8 \%$ for 10 seconds. Clear lysates were obtained after centrifugation for $5 \mathrm{~min}$ at $6000 \mathrm{rpm}$. Protein concentrations were determined using the $\mathrm{BCA}$ method (Molecular Probes). Equal amounts of protein were boiled for $5 \mathrm{~min}$ in 2x SDS sample buffer and subjected to 10 or $12 \%$ SDS-PAGE electrophoresis at $20 \mathrm{~mA} /$ gel before being transferred to a polyvinylidenedifluoride (PVDF) membrane, which had to be activated in methanol before. Transfer lasted $1 \mathrm{~h}$ and $30 \mathrm{~min}$ at $350 \mathrm{~mA}$ and was followed by blocking in $4 \%$ skimmed milk in $0.08 \%$ Tween 20/Tris-buffered saline (TBS-T) for $30 \mathrm{~min}$ prior to incubation with primary antibody. The dilutions for primary antibodies are described in Supplementary Table 1. After incubating the primary antibody overnight at $4{ }^{\circ} \mathrm{C}$ under gently shaking, membranes were washed three times with TBS-T for $10 \mathrm{~min}$ each and incubated with the appropriate horseradish peroxidase-conjugated secondary antibody for $1 \mathrm{~h}$ (antibody dilution 1:10,000) followed by the same washing procedure. Immunoreactive proteins were detected by enhanced chemiluminescence (Amersham Biosciences). Densitometric quantification of the band intensity was normalised to tubulin levels using Adobe Photoshop CS5. 


\section{EGFR endocytosis, starvation and degradation assay}

For EGFR endocytosis and degradation analysis, A431 cells were starved in DMEM without serum for $4 \mathrm{~h}$. After starvation, cells were treated with EGF $(100 \mathrm{mg} / \mathrm{ml})$ to stimulate EGFR endocytosis. Cells were then collected at various time points and lysed in RIPA buffer $(50 \mathrm{mM}$ Tris- $\mathrm{HCl} \mathrm{pH}$ 7.5, 1\% Triton X-100, $150 \mathrm{mM} \mathrm{NaCl}, 1 \mathrm{mM}$ EDTA and $0.1 \% \mathrm{Na}$ deoxycholate) containing protease inhibitor. Protein extracts were resolved by SDS-PAGE and immunoblotted using anti-EGFR antibody.

\section{EGFR surface biotinylation assay}

Surface biotinylation assays were performed as previously described ${ }^{35}$ with minor modifications. Briefly, transfected A431 cells were starved in DMEM without serum for $4 \mathrm{~h}$. After starvation, cells were treated with EGF to stimulate EGFR endocytosis and then preceded for surface biotinylation assays according to the manufacturer's protocol.

\section{Subcellular fractionation}

The subcellular distributions of distinct proteins were determined by using a subcellular fractionation kit following the manufacturer's protocol (Thermo Scientific/ Life Technologies) and analysed by immunoblotting. The subcellular fractionation was performed on HeLa cells as well as on NSC34 cells, both transfected with GFP-wtVAPB or P56S-VAPB.

\section{Immunohistochemistry Diaminobenzidine}

In all, 3-4- $\mu$ m paraffin sections were placed on poly-Llysine coated slides and allowed to dry in an oven $\left(37^{\circ} \mathrm{C}\right)$ overnight and then processed for immunohistochemistry described in detail elsewhere ${ }^{32}$. Sections deparaffinized in xylene for $20 \mathrm{~min}$ were rehydrated in $100 \%$, 95\% and $70 \%$ ethanol for $5 \mathrm{~min}$ each followed by endogenous peroxidase quenching $\left(0.3 \% \mathrm{H}_{2} \mathrm{O}_{2}\right.$ in methanol) for $20 \mathrm{~min}$. For antigen retrieval, sections were heated in citrate buffer, pH 6 (DAKO), for $20 \mathrm{~min}$ in a pressure cooker. After washing in PBS, sections were incubated with primary antibody (Supplementary Table 1) for $1 \mathrm{~h}$ at room temperature or at $4{ }^{\circ} \mathrm{C}$ overnight. After washing in PBS, sections were incubated with appropriate polymeric HRPlinker secondary antibody (IL Immunologic, Duiven, The Netherlands) for $30 \mathrm{~min}$ at room temperature. Diaminobenzidine (DAB) reagent (DCS Innovative Diagnostic System DAB kit) was used to stain the sections which were then counter-stained with $6 \%$ hematoxylin for $3 \mathrm{~min}$. All procedures were performed at room temperature. Standard histological and histochemical stains including H\&E, modified Gomori trichrome, non-specific esterase and NADH were performed as described by us and others previously $^{41}$.

\section{Immunofluorescence}

Single and double immunofluorescence staining was performed as described by us already elsewhere ${ }^{32,42}$. In brief, deparaffinized tissue sections were heated in citrate buffer, pH 6 (Dako), for $20 \mathrm{~min}$ in a pressure cooker. Sections were then blocked (to avoid non-specific bindings) with ready to use $10 \%$ normal goat serum (Life Technologies, MD, USA) for $1 \mathrm{~h}$ at room temperature before incubating with primary antibody at $4{ }^{\circ} \mathrm{C}$ overnight. After washing in TBS-T for $10 \mathrm{~min}$, the sections were incubated with Alexa conjugated secondary antibody (1: 500 in TBS-T) at room temperature for $2 \mathrm{~h}$. Sections were washed in TBS-T $(2 \times 10 \mathrm{~min})$ and stained for $10 \mathrm{~min}$ with $0.1 \%$ Sudan Black in $80 \%$ ethanol to suppress endogenous lipofuscin auto-fluorescence. Finally, the sections were washed for $5 \mathrm{~min}$ in TBST and mounted with Vectashield mounting medium (Vector Laboratories) containing DAPI.

\section{Transmission electron microscopy (TEM)}

NSC-34 cells were transfected with pEGFP-N1, GFPwt-VAPB or P56S-VAPB as described above. Cells were collected by scraping and then washed in $0.1 \mathrm{M}$ phosphate buffer and immediately fixed with $2.5 \%$ glutaraldehyde in $0.1 \mathrm{M}$ phosphate buffer for $24 \mathrm{~h}$ followed by washing in buffer for another $24 \mathrm{~h}$. Cell pellets were collected by centrifugation (1000 rpm, $5 \mathrm{~min}$ ) and embedded in $2 \%$ agarose (at $60^{\circ} \mathrm{C}$; Fluka \# 05073). Small blocks of embedded cells were sliced and post-fixed in $2.5 \%$ glutaraldehyde for $24 \mathrm{~h}$ followed by washing in $0.1 \mathrm{M}$ phosphate buffer for $24 \mathrm{~h}$. Agarose blocks were then incubated in $1 \% \mathrm{OsO}_{4}$ (in $0.2 \mathrm{M}$ phosphate buffer) for $3 \mathrm{~h}$, washed twice in distilled water and dehydrated using ascending alcohol concentrations (i.e. 25\%, 35\%, 50\%, 70\%, 85\%, 95\%, 100\%; each step for $5 \mathrm{~min}$ ). Dehydrated blocks were incubated in propylenoxide followed by subsequent 20 min incubation in a 1:1 mixture of epon $(47.5 \%$ glycidether, 26.5\% dodenylsuccinic acid anhydride, $24.5 \%$ methylnadic anhydride and $1.5 \%$ Tris (dimethylaminomethyl) phenol) and propylenoxide. The samples were then incubated in epoxy resin for $1 \mathrm{~h}$ at room temperature followed by polymerisation $\left(28^{\circ} \mathrm{C}\right.$ for $8 \mathrm{~h}, 80^{\circ} \mathrm{C}$ for $2.5 \mathrm{~h}$ and finally at room temperature for $4 \mathrm{~h}$ ). Ultra-thin sections $(70 \mathrm{~nm})$ were mounted on grids for electron microscopy and examined using a Philips CM10 transmission electron microscope as already described ${ }^{19}$.

\section{Image acquisition}

Images of the DAB-stained sections were taken with a Zeiss Axioplan microscope equipped with a 40x objective and an Axio Cam 506 color camera (Zeiss). Images from immunofluorescence labelled sections were taken with a Zeiss LSM 700 laser scanning confocal microscope using $\times 20, \times 40$ and $\times 63$ objectives. Images were acquired by 
averaging 4 scans per area of interest resulting in an image size of $1024 \times 1024$ pixels. The laser intensity was kept constant for all the samples examined. Captured confocal images were analysed using Adobe Photoshop CS5 and ZEN (Blue edition) 2009 software.

\section{Statistical analysis}

We used one-way ANOVA for comparison of multiple groups within individual experiments and also applied unpaired Student's $t$ test for comparison between two sample groups for the evaluation of significant differences using Graph pad Prism software. Values were expressed as mean \pm standard error of mean (SEM) from three independent experiments. For the FRAP and colocalisation experiments, we used the Mann-Whitney $U$ test. For both statistical tests, differences between the compared experimental conditions were regarded as significant when ${ }^{*} P<0.05$, ${ }^{* * * *} P<0.0001$, while \# denotes absence of a significant difference. For the quantification of numbers of autophagosomes (Fig. 2h), large, membrane-bound autophagosomes, filled with the cargoes were counted manually from EM images for 20-30 cells from both wt-VAPB as well as P56S-VAPB cells. Sample sizes of all experiments were determined based upon our experience. No sample was excluded from the analyses. Animals were carefully assigned during collection not randomly, based upon same strain, sex, and age. The replicates numbers are indicated in each figure legend.

\section{Results}

\section{P56S-VAPB leads to defective autophagy in ALS8}

Over expressed P56S-VAPB forms ER-associated aggregates in cells, hiPSC MNs (Supplementary Fig. 1a, b) as well as in the P56S-VAPB transgenic mouse models (Supplementary Fig. 1c); in contrast, P56S-VAPB inclusions were absent in induced pluripotent stem cell (iPSC)derived MNs of ALS8 patients ${ }^{43}$. Autopsy tissues from ALS8 patients are not available so far to address the toxic gain of function of P56S-VAPB, however, for current study, we could use muscle biopsies obtained from an index ALS8 patient of European origin ${ }^{5}$ and from his affected mother. H\&E staining of the index patients' muscle biopsy and the routine histology from his affected mother showed severe neurogenic grouped fibre atrophy (Fig. 1a and Supplementary Fig. 1d), associated with increased immunoreactivity for several ER chaperones and proteotoxicity markers (Supplementary Fig. 1e) in partially atrophic and atrophic muscle fibres of the index patient.

Using our custom made VAPB antisera ${ }^{31-33}$, we found accumulation of globular aggregates of VAPB in denervated muscle fibres (Fig. 1b, arrowheads). Normal-sized innervated muscle fibres in this biopsy as well as control biopsies showed mild to moderate sarcoplasmic VAPB immunoreactivity (Fig. 1b). Skin fibroblasts obtained from this ALS8 patient revealed smaller prominent punctate VAPB aggregates (Fig. 1c), which formed larger globular aggregates when challenged with proteasome or autophagy inhibitors (Fig. 1c; arrowheads right panel). Western blot analysis confirmed the presence of SDS-insoluble gel top VAPB aggregates in ALS8 fibroblast (Supplementary Fig. 1f)

Since VAPB protein is crucial for autophagy ${ }^{14,20}$, we used the ALS8 muscle biopsy and the patient-derived fibroblasts to understand if aggregated P56S-VAPB affects the autophagy pathway.

DAB immunohistochemistry using antibodies against autophagic markers LC3 and p62 revealed their accumulations in degenerating fibres containing large autophagic vacuoles (Fig. 1d; arrows). Immunofluorescence showed even more prominent accumulations of large LC3 and p62 puncta, which were specifically co-localised with globular VAPB aggregates (Fig. 1e). Consistent with these findings, ALS8 fibroblasts also showed co-localisation of accumulated LC3 and p62 together with P56S-VAPB aggregates (Fig. 1f; arrowheads).

P56S-VAPB aggregates sequestering both LC3 and p62 were indicative of altered autophagy. Thus we next analysed the autophagy status in several cell lines expressing P56S-VAPB tagged with GFP following current guidelines ${ }^{44-47}$. Western blot analysis showed LC3 II and p62 were elevated together with the ER stress markers GRP78 both in P56S-VAPB transfected cells (Fig. 1g and Supplementary Fig. 1g) and in ALS8 fibroblasts (Supplementary Fig. 1f). Interestingly, consistent with the appearance of SDS insoluble VAPB smears (black arrowhead, Fig. 1g) in P56S-VAPB expressing cells, p62 protein was also aggregated at the gel top (red arrowhead, Fig. 1g). In line with this LC3 and p62 positive autophagosomes were found to be abnormally accumulated and sequestered with P56S-VAPB aggregates (Fig. 1h, i and Supplementary Fig. 1h, i). Similarly, a consistent pattern of accumulated LC3 by immunofluorescence analysis (Fig. 1j), confirmed by Western blot studies (Fig. 1k) was observed in NIH3T3 fibroblasts stably expressing GFP$\mathrm{LC}^{35,42}$. Furthermore, we applied a widely used method to analyse the internalization and degradation of the EGFEGFR complex in A431 cells that express high EGFR levels which is exclusively degraded via autophagy ${ }^{35,42,48}$. Here again, expression of P56S-VAPB led to the accumulations of EGFR together with p62 and LC3 (Fig. 1l). The expression of wt or P56S- VAPB did not impair EGFR internalization after $10 \mathrm{~min}$ of EGF stimulation (not shown). However, P56S-VAPB, in contrast to wt-VAPB, impeded time-dependent degradation of EGFR (Fig. 1m). Defective autophagy was further characterised by ultrastructural analysis of P56S-VAPB-transfected cells, 


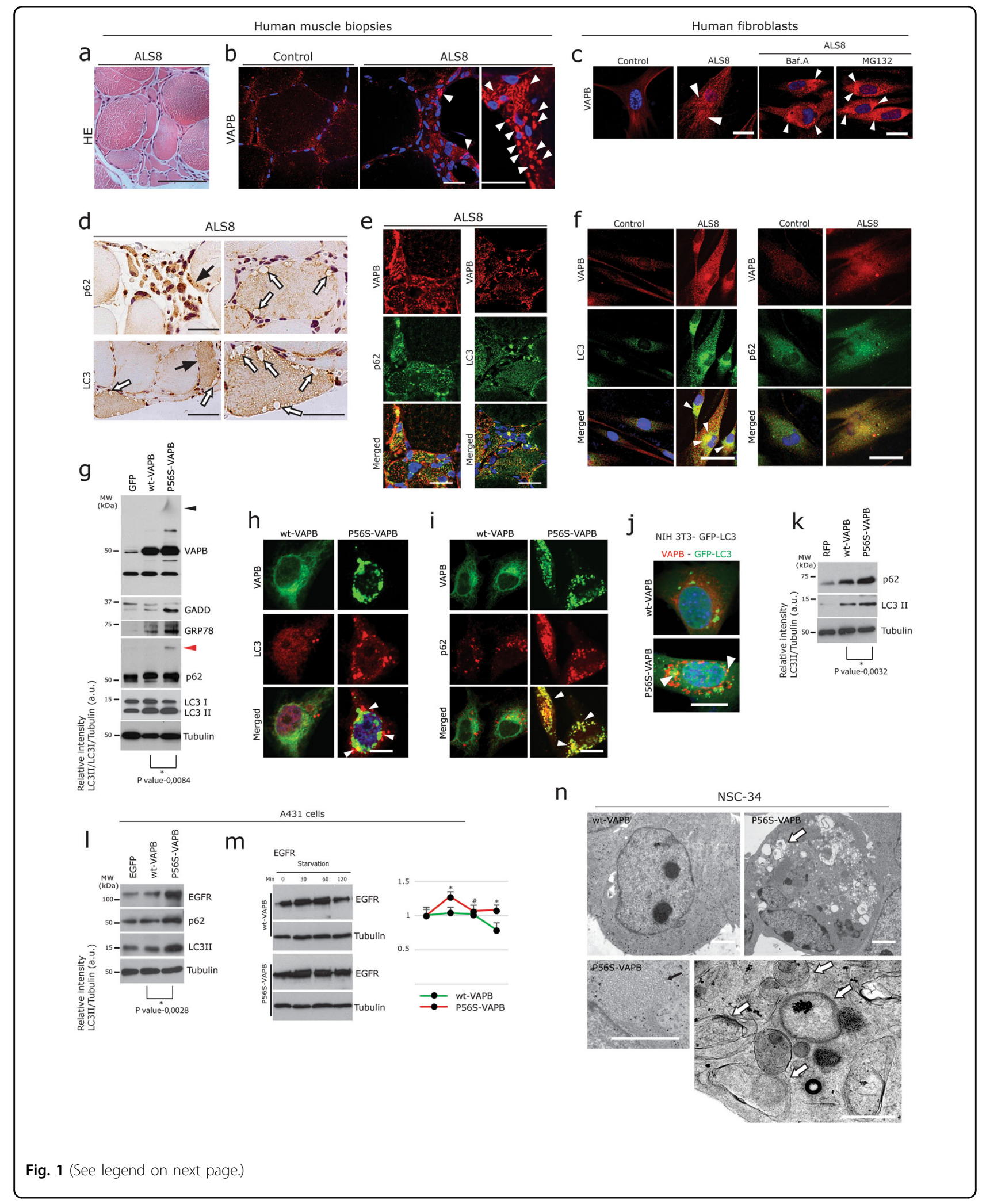


(see figure on previous page)

Fig. 1 P56S-VAPB leads to defective autophagy in ALS8. a Representative H\&E stained section from ALS8 index patient muscle biopsy showing typical neurogenic atrophy; presence of angular, flatten and group fibre atrophy. Scale bar: $60 \mu \mathrm{m}$. b Prominent immunoreactivity of globular VAPB aggregates (arrowheads, right panels) in grouped angular or flattened, atrophic, denervated muscle fibres of ALS8 compared to the normal control (left panel) showing diffuse pattern of sarcomeric VAPB immunolabelling in normal-sized fibres. Scale bars: $70 \mu m$. c Immunolabeling of human fibroblasts using VAPB antibody showing small but enormous punctate P56S-VAPB aggregates in ALS8 fibroblast (right panel, white arrowheads), compared to control (left panel). Note the increased formation of globular P56S-VAPB aggregates (arrowheads) in ALS8 fibroblasts, after treatment with proteasome inhibitor MG132 $(2 \mu \mathrm{M})$ and Bafilomycin A $(200 \mathrm{nM})$ for $4 \mathrm{~h}$. Scale bars: $10 \mu \mathrm{m}$. d DAB immunohistochemistry performed on ALS8 muscle biopsy showing increased accumulation of autophagy marker p62 and LC3 in atrophic and partially atrophic fibres. Note the numerous large autophagic vacuoles (white arrows) present in hypertrophic fibres. Scale bars: $50 \mu \mathrm{m}$. e Double immunofluorescence labelling of ALS8 muscle biopsy using antibodies against VAPB and for the autophagy marker p62 and LC3 showing co-localisation of the aggregated p62 and LC3 together with the mutant VAPB aggregates. Scale bars: $25 \mu \mathrm{m}$. f Punctate P56S-VAPB aggregates co-localised with LC3 and p62 accumulations in ALS8 fibroblasts. Scale bars: $10 \mu \mathrm{m} . \mathbf{g}$ Immunoblot analysis in NSC-34 cells transiently transfected either with empty GFP vector, wt-VAPB and P56S-VAPB. Note the increased levels of ER stress, autophagy markers as well as presence of gel top smear aggregate of P56S-VAPB (black arrowhead) and aggregated p62 (red arrowhead) in P56S-VAPB expressing cells. Corresponding densitometric data are shown at the bottom; representing the relative band intensity of LC3-II/LC3-I, normalised with tubulin levels. Graph pad prism, unpaired Student's $t$ test for comparison between two sample groups. Values were expressed as mean \pm standard error of mean (SEM) from three independent experiments. The asterisks denote significant differences $\left({ }^{*} p<0.05\right)$. $\mathbf{h}, \mathbf{i}$ Co-labelling of wt-VAPB and P56S-VAPB with LC3 (h) and with p62 (i). Note that the globular accumulations of both LC3 and p62 and their colocalisation with P56S aggregates. Scale bar: $10 \mu \mathrm{m}$. j, k Increased accumulation of autophagosomes (white arrowheads) in the stable autophagy reporter cell line NIH3T3-GFP transfected with P56S-VAPB. Green: GFP-LC3; red: VAPB; scale bar: $10 \mu \mathrm{m}$ (j). Immunoblot analysis showing increased levels of both p62 and LC3 in cells transfected with P56S-VAPB (k). Unpaired Student's $t$ test for comparison between two sample groups. Values were expressed as mean \pm standard error of mean (SEM) from three independent experiments. The asterisks denote significant differences $\left({ }^{*} p<0.05\right)$. I Immunoblot analysis of A431 cells transfected with EGFP, wt-VAPB and P56S-VAPB showing the increased levels of the autophagy markers EGFR, p62 and LC3 in P56S-VAPB expressing cells. Unpaired Student's t-test for comparison between two sample groups. Values were expressed as mean \pm standard error of mean (SEM) from three independent experiments. The asterisks denote significant differences $\left({ }^{*} p<0.05\right)$. $\mathbf{m}$ A431 cells were transfected as described above. $24 \mathrm{~h}$ later, transfected cells were processed for the EGFR degradation assay (see Methods) and analysed by immunoblotting with the EGFR antibody. Note the delayed EGFR degradation in P56S-VAPB expressing cells. Right panel: quantification of immunoblots analysis. Graph pad prism, unpaired Student's $t$ test for comparison between two sample groups. Values were expressed as mean \pm standard error of mean (SEM) from three independent experiments. The asterisks denote significant differences $\left({ }^{*} p<0.05\right)$, while \# denotes absence of a significant difference. $\mathbf{n}$ NSC-34 cells overexpressing wt-VAPB and P56S-VAPB were fixed with $2.5 \%$ buffered glutaraldehyde and processed for EM. Several membrane-bound vacuolar structures (white arrows) often containing cargoes and probably derived from the ER in a representative P56S-VAPB-expressing cell compared to cells expressing wt-VAPB control. Higher magnification showing the ER membrane proliferation into tubular structures (black arrow; lower left panel), and large autophagic vacuoles often filled with cargoes (white arrow; lower panel) in representative P56SVAPB expressing cells. Scale bars: $1 \mu \mathrm{m}$ (upper panel), $0.5 \mu \mathrm{m}$ (lower panel).

showing prominent accumulations of numerous autophagic vacuoles (white arrows) which also sequestered cellular components/cargoes (Fig. 1n). These changes were accompanied by the cytoplasmic accumulation of tubular structures (black arrow), probably reflecting ER proliferation (Fig. 1n).

\section{P56S-VAPB alters both early and late steps of autophagy}

Aggregated p62 at the gel top, co-localisation of LC3 and p62 with P56S-VAPB aggregates and increased LC3II in Western blots (Fig. 1) were all indicative of blocked autophagic flux. However, to further rule out that these effects were not due to increased autophagic activity, we next systematically monitored autophagic flux ${ }^{45,47}$. Compromised degradation of accumulated LC3II due to P56S-VAPB was confirmed by Western blot analysis in NSC-34 cells, where autophagy induction (Rapamycin) or inhibition (Bafilomycin A) only marginally, if at all, affected p62 and LC3-II levels in P56S-VAPB expressing cells compared to wt-VAPB (Fig. 2a). Consistently in GFP-LC3 reporter lines and in primary mouse embryonic fibroblasts, the absence of a significant increase in LC3-II and p62 protein levels even after Bafilomycin A treatment
(Fig. 2b, c) further confirmed impaired clearance of accumulated autophagosomes.

A possible scenario underlying the above phenomenon might be the fusion defects of autophagosomes to lysosomes in P56S-VAPB expressing cells. To test this possibility, we monitored the fusion of autophagosomes to lysosomes using previously described NIH3T3- mCherryGFP-LC3 cells ${ }^{49}$. As the GFP signal is lost upon degradation in the acidic environment of lysosomes, loss of GFP signal relative to mCherry signal is a read out of autophagosome fusion with lysosomes ${ }^{49}$. Consistent with this notion, live cell imaging showed that GFP signal was relatively preserved in mCherry-GFP-LC3 cells that also expressed P56S-VAPB, suggesting that the fusion of autophagosomes with lysosomes was impaired by P56SVAPB (Fig. 2d, e). In contrast, we observed both a clear reduction of GFP fluorescence and RFP-GFP co-localisation in nearly all wt-VAPB expressing cells (Fig. 2d, e). To further support this conclusion, we analysed the SNARE protein syntaxin 17 (STX17) which is essential for the fusion of lipid bilayers and required for autophagosomelysosome fusion ${ }^{50}$. Using Immunofluorescence analysis, we observed that STX17 protein immunolabelling were 


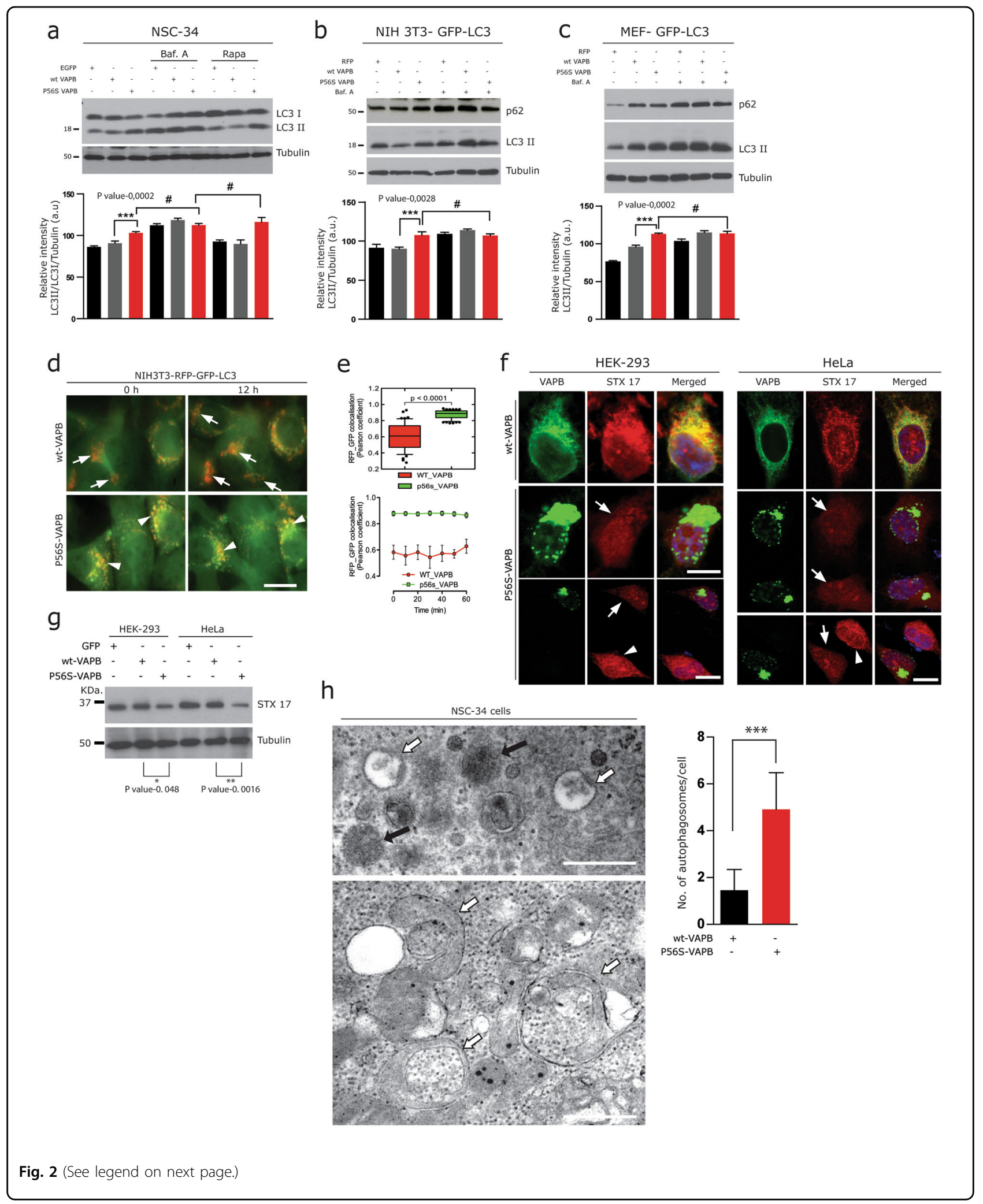


(see figure on previous page)

Fig. 2 P56S-VAPB alters late step of autophagy. a-c Immunoblot analysis of transiently transfected control GFP/RFP, wt-VAPB and P56S-VAPB; in NSC-34 cells (a), NIH3T3-GFP-LC3 fibroblasts (b) and of the Primary fibroblasts isolated from autophagy reporter GFP-LC3 transgenic mice (MEF-GFPLC3) (c), and then additionally treated with the autophagy inhibitor Bafilomycin A ( $2 \mu \mathrm{M})$ for $2 \mathrm{~h}$ to inhibit autophagy followed by treatment of Rapamycin $(2 \mu \mathrm{M})$ for $4 \mathrm{~h}$ to accelerate autophagy (a) or Bafilomycin A $(2 \mu \mathrm{M})$ alone $(\mathbf{b}$, $\mathbf{c})$. Note the unchanged LC3-II levels in P56S-VAPB transfected cells after Rapamycin or Bafilomycin A treatment. Corresponding densitometric data are shown at the bottom; where the numbers represent the relative band intensity of LC3-II/LC3-I, normalised with tubulin levels. Graph pad prism, unpaired Student's $t$ test for comparison between two sample groups. Values were expressed as mean \pm standard error of mean (SEM) from three independent experiments. The asterisks denote significant differences $\left({ }^{*} p<0.05,{ }^{* * *} p<0.001\right.$ ), while \# denotes absence of a significant difference. d, e NIH3T3 fibroblasts stably expressing RFP-GFP-LC3 was transfected either with HA tagged wt-VAPB or P56S-VAPB. In all, $24 \mathrm{~h}$ later the fusion of autophagosomes with lysosomes was measured by live cell imaging for additional $12 \mathrm{~h}$ (d), the rate of autophagosome fusion reflected by the Pearson coefficient (green/red fluorescence ratio) at each time point indicated (e). Values are represented as means \pm S.E.M. of triplicate experiments ${ }^{*} p \ll 0.0001$. $\mathbf{f}$ Co-labelling of wt-VAPB and P56S-VAPB transfected HEK-293 as well as HeLa cells with STX17, showing its marked reduction (arrows) in cells harbouring P56S-VAPB aggregates. Scale bar: 10 mm. $\mathbf{g}$ Immunoblot analysis of STX17 proteins in HEK-293 as well as HeLa cells, transiently transfected with control GFP, wt-VAPB and P56S-VAPB. Note the significantly reduced levels of STX17 in P56S-VAPB overexpressing cells. Unpaired Student's $t$ test for comparison between two sample groups. Values were expressed as mean \pm standard error of mean (SEM) from three independent experiments. The asterisks denote significant differences ( $\left.{ }^{*} p<0.05\right)$. $\mathbf{h}$ EM analysis of P56S-VAPB transfected NSC-34 cells showing massive accumulation of autophagosomes (white arrows, quantification) in the vicinity of several lysosomes (black arrows). For the quantification of autophagosomes, numbers of large, membrane-bound autophagosomes, filled with the cargoes were counted manually from 20 to 30 cells from both the wt-VAPB as well as P56S-VAPB. The asterisks denote significant differences $\left({ }^{* *} p<0.001\right)$. Scale bars: $1 \mu \mathrm{m}$.

markedly reduced in HEK-293 as well as in HeLa cells harbouring P56S-VAPB aggregates (Fig. 2f, arrows). Consistent with this findings Western blot analysis of the above cell lysates showed a significant decrease in STX17 protein levels in P56S-VAPB overexpressing cells compared to the wt-VAPB and control GFP overexpressing cells (Fig. 2g, quantification below).

Finally, electron microscopy performed on NSC-34 cells revealed that, consistent with impaired fusion, massive accumulation of autophagosomes with cargoes (white arrows) were found in the vicinity of lysosomes (black arrows), indicative of impairment of their fusion (Fig. 2h, quantification of the accumulated autophagosomes). Taken together, our findings demonstrate that P56SVAPB impairs the autophagic degradation due to incomplete clearance of autophagosomes (i.e., late-stage autophagy).

Recently, Zhao et al. reported that P56S-VAPB mutation reduces the ULK1/FIP200 interaction and impairs autophagy at an early stage, namely autophagosome biogenesis $^{14}$. Therefore, as a next logical step, we also analysed the effect of P56S-VAPB on early steps of autophagy. We focussed on ULK1 as well as on ATG5ATG12, because the ATG5- ATG12 containing complex is essential for membrane expansion and completion of the autophagosome $\mathrm{e}^{51-53}$

While both ULK1 and ATG5-ATG12 proteins were distributed throughout the cell, largely co-distributing with wt-VAPB (Fig. 3a, b); however, they both sequestered with P56S-VAPB aggregates in P56S-VAPB expressing cells (Fig. 3a, b arrows). Filter trap assays further confirms the presence of SDS resistant aggregates of both of these proteins in P56S-VAPB expressing cells (Fig. 3c). Consistent with this, Western blots indicated that ULK1 protein levels were elevated in wt-VAPB expressing cells (Fig. 3d), whereas in P56S-VAPB expressing cells, fractions of ULK1 protein remained at the gel-top as an aggregated form (Fig. 3d, e; red arrowhead), thus resulting in reduced amounts of its soluble form (Fig. 3d, e). Similar to ULK1 and consistent with the filter trap assay, ATG5ATG12 protein complex (actual molecular weight $=$ $55 \mathrm{kDa}$ ) was also found to be aggregated at the gel-top (Fig. 3d). Even though such alterations of crucial autophagy-associated proteins are consistent with defects in early phases of autophagy ${ }^{14}$; increased levels of LC3-II in the same samples (Fig. 3d, e) together with our earlier observations (Fig. 2) suggest defects in late-stage autophagy. We used multiple cell lines expressing either the wt or P56S-VAPB (Figs. 2, 3d-g, and Supplementary Fig. 1j) and in most of them P56S-VAPB induced both the reduction of soluble form / aggregation of ULK1 at the gel top as well as increased levels of p62/LC3-II. Based on these observations, we concluded that P56S-VAPB alters both late and early steps of autophagy simultaneously.

\section{Autophagy defects in P56S-VAPB transgenic mice}

Prompted by the above findings we next examined autophagy markers in transgenic mice expressing P56Smutant human $V A P B$ that develop VAPB aggregates both in lumbar spinal cord $\alpha-\mathrm{MNs}$ as well as in cortical neurons accompanied by ER stress ${ }^{15,22}$ (Fig. 4a and Supplementary Fig. 2c-f). Both lumbar spinal cord $\alpha$-MNs (Fig. 4b, c) and cortical neurons (Supplementary Fig. 2e) consistently showed co-localisation of LC3 and p62 together with globular P56S-VAPB aggregates. In line with the above observations in cell culture models (Figs. 2 and 3 ), we also found significantly reduced immunoreactivity of STX17 (Fig. 4e) and sequestration of early 


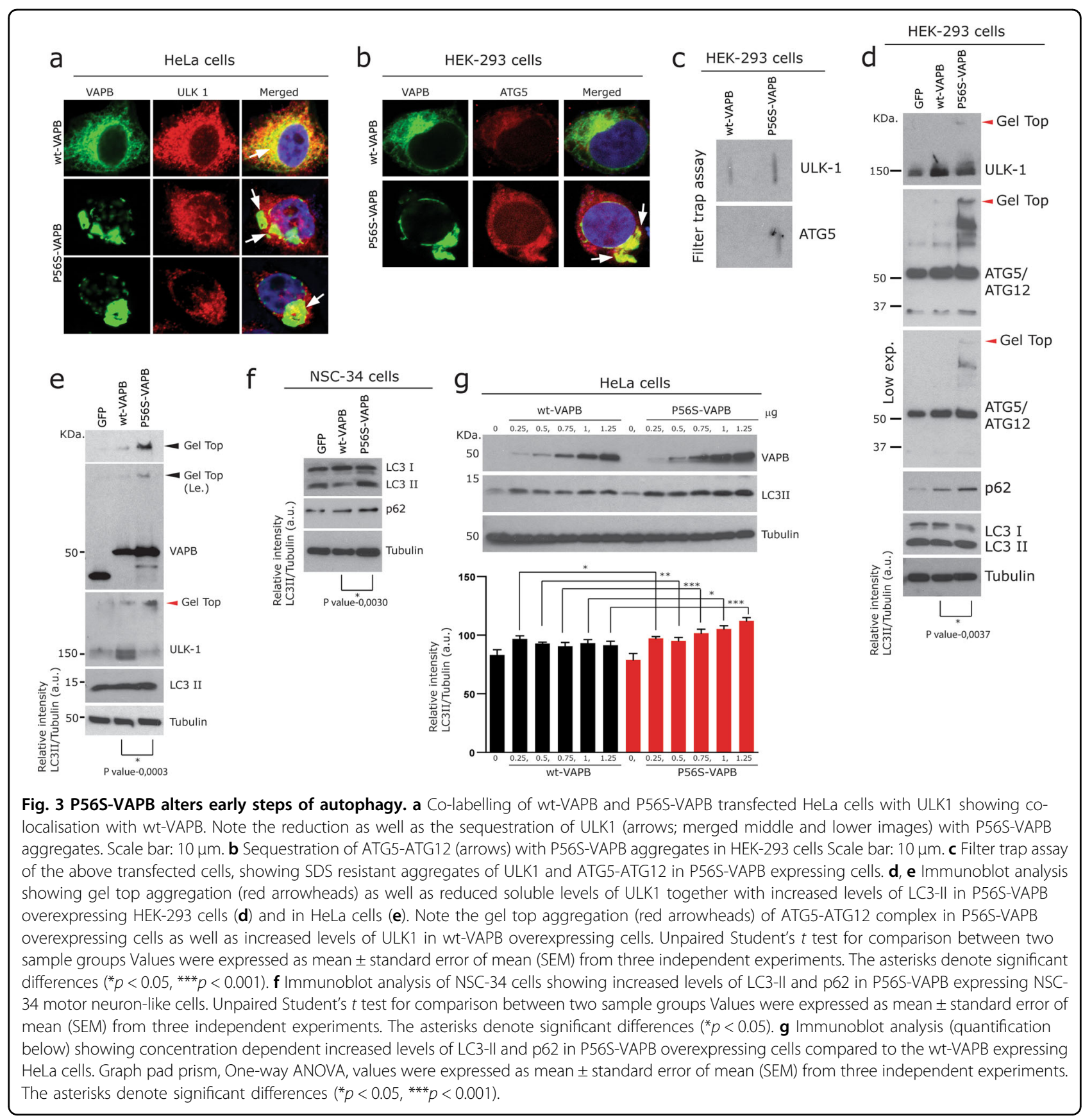

autophagy proteins ULK1, ATG5-ATG12 together with the globular P56S-VAPB aggregates in the lumbar spinal cord $\alpha$-MNs of P56S-VAPB mice (Fig. 4f, g). Consistent with this, Western blot analysis of lumbar spinal cord lysates and the total brain lysates revealed increased levels of p62 as well as LC3 together with markedly increased UPR (Fig. 4d and Supplementary Fig. 2h). Interestingly, here again p62 appeared as SDS-insoluble aggregates at the gel-top in the immunoblots of total brain lysates of these mice (Supplementary Fig. $2 \mathrm{~h}$, red arrowhead). These in vivo results together with the cell culture observations establish that autophagy is altered at multiple stages, leading to neuronal and axonal degeneration of both cortical as well as spinal cord $\alpha$-MNs (Supplementary Fig. $2 a, f, g)$.

\section{P56S-VAPB aggregation leads to altered distribution of other fALS-associated RBP}

Efficient turnover of several fALS-associated RBPs such as TDP-43, FUS and of other RNA SGs is regulated by UPS and autophagy ${ }^{54,55}$. Similarly, alterations of RBP homeostasis can directly affect proteostasis (reviewed in 


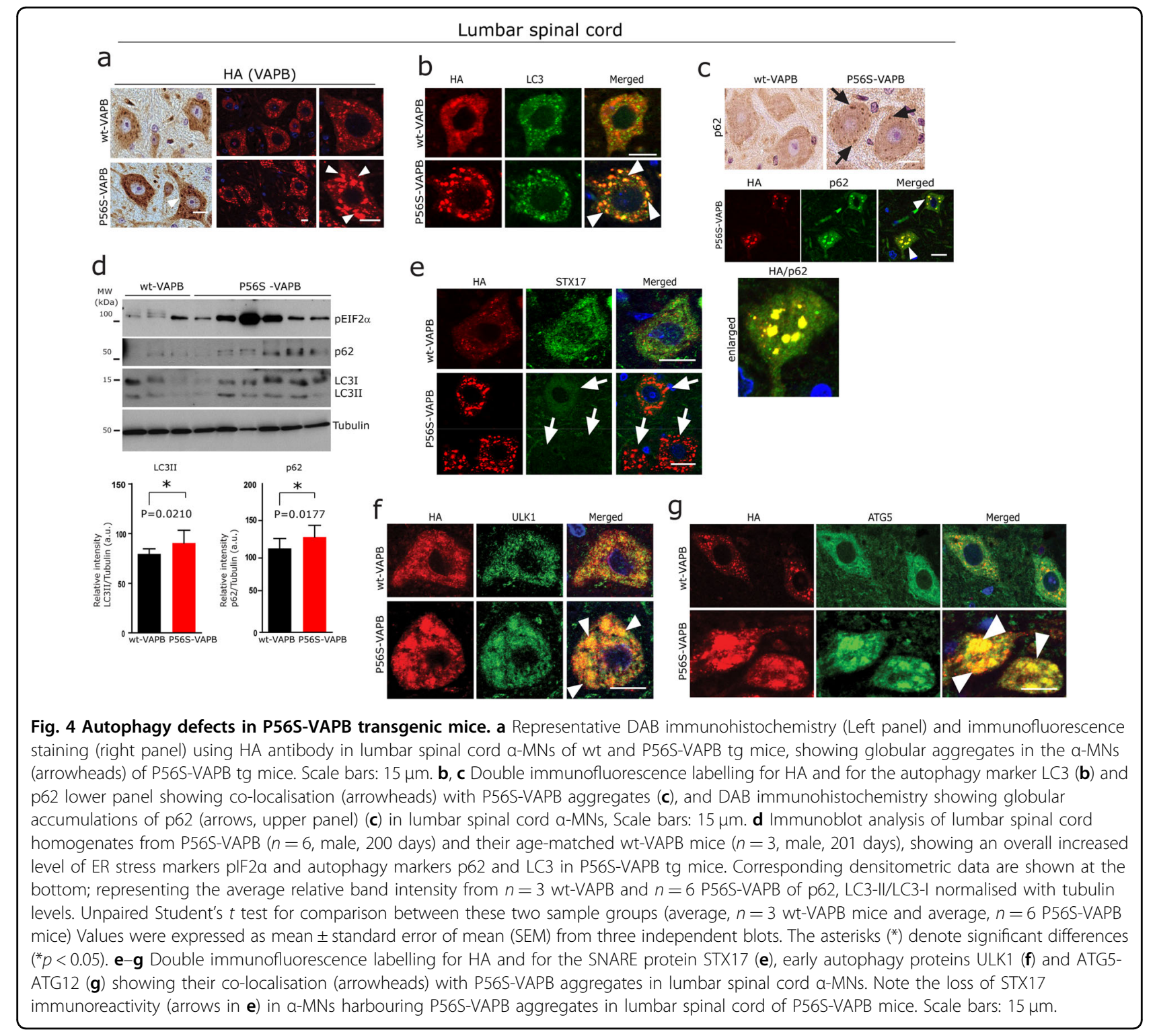

Walsh et al..$\left.^{56}\right)$. Since P56S-VAPB aggregates were associated with impaired autophagy, we asked whether P56SVAPB-mediated toxicity is also linked to the altered distribution RBPs, in particular the key ALS proteins TDP43, FUS and Matrin 3. These proteins normally reside in the nucleus; in ALS, they often accumulate in the cytoplasm of affected neurons ${ }^{1,26,29}$. We found that P56S-VAPB aggregates are associated with loss of nuclear localisation and cytoplasmic accumulation of above mentioned RBPs (Fig. 5a-c) compared to the wt-VAPB transfected cells, where they remained in the nucleus (Fig. 5a-c; upper panels). In many instances, P56S-VAPB was partially colocalised with some of these cytoplasmic RBP aggregates (Fig. 5a-c). In line with this, subcellular fractionation performed on P56S-VAPB expressing cells further confirmed the elevated protein levels of TDP-43, FUS and
Matrin 3 in the cytoplasmic fractions (Fig. 5d, red arrowheads). Accordingly, there was mild to moderate loss of nuclear localisation combined with diffuse cytoplasmic accumulation of pTDP-43 and Matrin 3 in $\alpha$ MNs of P56S-VAPB compared to wt-VAPB Tg mice (Fig. 5e). Even though cytoplasmic FUS accumulations were rather rare, loss of nuclear FUS was prominent in the $\alpha-$ MNs of the P56S-VAPB mice (Fig. 5f). Prompted by these observations, we further examined the ALS8 patient muscle biopsy. Similar to the characteristic pTDP-43 aggregates found in sporadic and familial ALS $\alpha$-MNs, degenerating muscle fibres in this muscle biopsy displayed accumulations of large globular pTDP-43 aggregates (Fig. 5g). pTDP-43 aggregates can actually be seen in atrophic and partially atrophic fibres of many sALS and fALS autopsy $\operatorname{cases}^{57}$ (Supplementary Fig. 3d). 


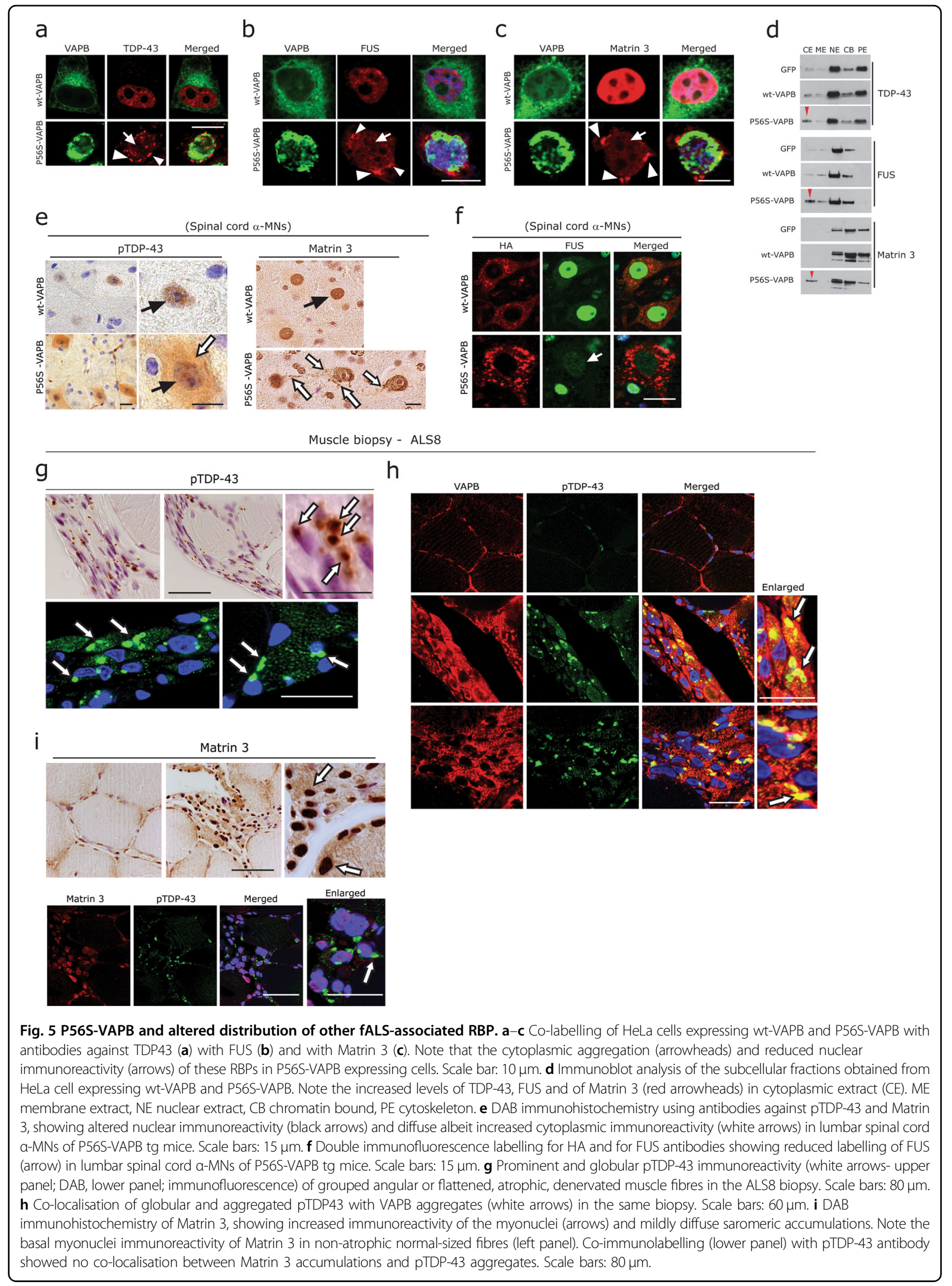


Interestingly, these abundant pTDP-43 aggregates were also found to be co-localised with globular VAPB aggregates (Fig. 5h, arrows, right panels). As expected, Matrin 3 immunoreactivity of myonuclei of degenerating muscle fibres were elevated (Fig. 5i), while the normal-sized fibres showed mild labelling of the myonuclei (Fig. 5i; left panel). A mild diffuse pattern of cytoplasmic Matrin 3 immunoreactivity was also observed in many degenerating fibres (Fig. 5i); however, there were no obvious cytoplasmic aggregates of Matrin 3 in this biopsy and there was no co-localisation with pTDP-43 aggregates (Fig. 5i).

\section{P56S-VAPB accumulation leads to abnormal stress granule formation}

Since aggregation of the above mentioned RBPs mostly proceeds through the SG pathway,26,29,58,59, we next examined SG formation in cells transfected with wt or P56S-VAPB. Consistent with the mis-localisations of many fALS-associated RBPs, we observed cytoplasmic accumulation of TIAR-1 and G3BP SGs in P56S-VAPB expressing cells (Fig. 6a, b). For further validations, we used a well-studied cell line stably expressing the SG protein $\mathrm{G}_{3 B \mathrm{P}^{38}}$. We found that SG formation in P56SVAPB transfected cells started within $10 \mathrm{~h}$, even before P56S-VAPB aggregates were formed (15 h after transfection). These aggregates increased in number and size at 24 h (Fig. 6c, d). We could not observe any SG formation in cells expressing wt-VAPB during the same period of time, however at later time $(36 \mathrm{~h})$ we could observe minor SGs in few cells (Fig. 6d). Considering that SGs are highly dynamic structures and that persistent stress and misfolded protein aggregation lead to prolonged SG stability and aggregation ${ }^{38,58,60}$, we next studied the dynamic properties of SGs formed by P56S-VAPB using fluorescence recovery after photobleaching (FRAP) analysis to measures the relative kinetics of SGs over the time course of the experiment ${ }^{38,58,60}$. FRAP analysis of these G3BPSGs showed a slower recovery in P56S-VAPB expressing cells compared rare SGs observed in cells expressing wtVAPB (Fig. 6e, f). The persistent accumulations of these SGs were further confirmed by subcellular fractionation showing that the SG protein TIAR1 accumulated in the cytoplasmic fraction (Fig. 6g) in P56S-VAPB expressing cells. In line with these cell culture observations, we observed an increased albeit diffuse immunoreactivity of TIAR1 in lumbar spinal cords $\alpha$-MNs of P56S Tg mice (Fig. 6h). Finally, we observed an enhanced immunoreactivity of large granular TIAR-1-positive SGs in ALS8 patient muscle biopsy (Fig. 6i, arrows). Interestingly, these SGs were co-localised with VAPB aggregates (Fig. 6j, arrowheads). In summary, consistent with the deregulation of RBPs, the robust formation of SGs is a predominant feature of P56S-VAPB-driven pathology.

\section{Discussion}

Altered RBPs homeostasis and defective proteostasis are key mechanisms triggering neurodegeneration in $\mathrm{ALS}^{1,26}$. In the present study we asked if P56S-VAPB is involved in these processes to initiates pathological cascades leading to neurodegeneration.

We found accumulations of $\mathrm{p} 62$ and LC3 protein and of their sequestration with P56S-VAPB aggregates in various cell culture models, P56S-VAPB mouse, ALS8 patient fibroblast and muscle biopsy (Figs. 1-4). Furthermore, alterations in autophagy flux were confirmed in multiple cell lines (Figs. 1-3). Consistent with this, we found numerous autophagic vacuoles associated with ubiquitinpositive accumulations in ALS8 patient muscle biopsy (Fig. 1). These results are in line with recent reports of P56S-VAPB accumulations in the autophagosome and with the ubiquitin-positive inclusions in MNs of ALS8 knock-in mice that were accompanied by increased levels of P62/SQSTM1 and $\mathrm{LC}^{24}$. Of note in line with the recently described role of VAPB in ER-phagy ${ }^{61}$, we found proliferated, expanded ER both in P56S-expressing cells and in the P56S-VAPB patient muscle biopsy (Fig. 1 and Supplementary Fig. 3e). Thus, it appears reasonable to propose that ER-assisted autophagy dysfunction is an early, central step of P56S-VAPB-mediated ALS pathology. In this context it is important to mention that apart from VAPB, mutations, several ER proteins including SigR1, SIL1, HSPB1, HSPB8 and HSJ1 cause defects in ER structure accompanied by autophagy impairment and lead to familial neurodegenerative disorders including motor neuron diseases ${ }^{62-68}$. In sALS, the above wild-type proteins accumulate in surviving MNs in sALS and might serve protective functions, as has been shown for SigR1 and SIL1 $1^{19,69,70}$.

VAPB interacts with ATG proteins and also facilitate the recruitment and stabilisation of ULK1 and FIP200 puncta at the ER, which is crucial for autophagosome formation ${ }^{14}$. Loss of VAPB and PTPIP51 (ER-mitochondria tethering complex) loosens the ER-mitochondria contacts and induces autophagic flux, whereas overexpression of wt-VAPB and PTPIP51 tightens the ERmitochondria contacts and thus impairs autophagy ${ }^{20,21}$. Thus, confirming normal VAPB is decisive for autophagy process. Consistent with these reports, reduction of ULK1 protein levels as well as co-aggregation of ULK1 and ATG5-ATG12 together with P56S-VAPB aggregates depicted in Fig. 3 and in $\alpha$-MNs of P56S-VAPB mice (Fig. 4) suggests probably an alteration of the initial phase of autophagy. However, the concomitant appearance of significantly increased levels of LC3-II in multiple cell lines overexpressing P56S-VAPB and detailed analysis of autophagic flux using autophagy reporter cell lines confirmed that P56S-VAPB also impairs the fusion of autophagosomes to lysosomes, which is a late autophagy 


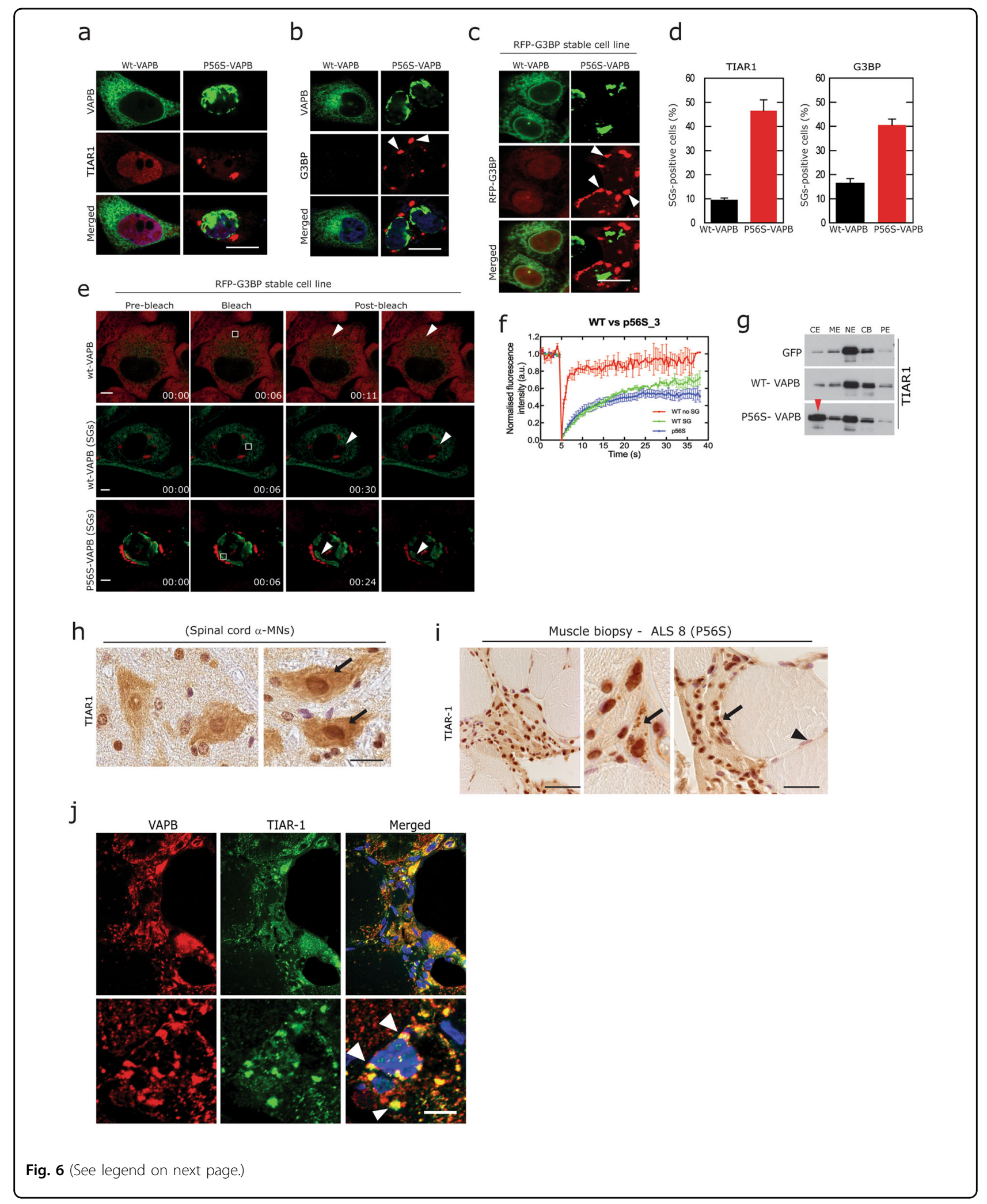


(see figure on previous page)

Fig. 6 P56S-VAPB leads to abnormal stress granules formation and alters their dynamics. $\mathbf{a}$, $\mathbf{b}$ Co-labelling of HeLa cells expressing wt-VAPB and P56S-VAPB with antibodies against SG markers TIAR1 (a) and G3BP (b), showing cytoplasmic SG formation (arrowheads) in P56S-VAPB expressing cells. Scale bar: $10 \mu \mathrm{m}$. c Immunofluorescence analysis of HeLa cells stably expressing SG protein G3BP, showing SG formation in P56S-VAPB transfected cells compared to wt-VAPB transfected cells after $24 \mathrm{~h}$. Scale bar: $10 \mu \mathrm{m}$. d Quantification of TIAR1 in HeLa cells and G3BP stable cell lines for SGs formation due to overexpression of either the wt-VAPB or P56S-VAPB after $36 \mathrm{~h}$ of transfection. e, $\mathbf{f}$ FRAP analysis by live cell imaging on the above cell lines showing reduced recovery of G3BP-SGs in P56S-VAPB transfected cells compared to wt-VAPB transfected cells, Quantifications (f). Scale bar: $10 \mu \mathrm{m}$. g Immunoblot analysis subcellular fractions of HeLa cells expressing wt-VAPB and P56S-VAPB, showing increased levels of TIAR1 in cytoplasmic fraction (red arrowhead, CE) in P56S-VAPB expressing cells. h, i DAB immunohistochemistry using antibodies against SG marker TIAR1 in lumbar spinal cord a-MNs of wt and P56S-VAPB Tg mice (h) and in ALS8 muscle biopsy (i), showing altered nuclear immunoreactivity (arrows) and diffuse albeit increased cytoplasmic immunoreactivity (arrows) in both a-MNs of P56S- VAPB tg mice as well as in ALS8 muscle biopsy. (Scale bars: $15 \mu \mathrm{m}$ in $\mathbf{h}, 60 \mu \mathrm{m}$ in $\mathbf{i}$. j Accumulated globular immunoreactive TIAR1 aggregates co-localises with VAPB aggregates (arrowheads) in ALS8 muscle biopsy. Scale bars: $25 \mu \mathrm{m}$.

step (Figs. 2 and 3). In this context it is important to note that besides orchestrating initial steps of autophagy, ULK1 can also regulate late steps of autophagy by promoting autophagosome to lysosome fusion ${ }^{71}$.

RBPs including many SGs are particularly susceptible to aggregation due to the presence of both RNA-binding domains and prion-like domains, which contribute strongly to aggregate formation under stressful stimuli including chronic autophagy impairment ${ }^{72,73}$. In addition, loss of dynamic nature of these SGs together with their impaired clearance could results in the formation of aberrant and persistent pathological inclusions ${ }^{2,38,58,74-76}$. Notably, P56S-VAPB mice show signs of TDP-43 pathology ${ }^{23}$. Furthermore, the expression of P56S-VAPB enhanced TDP-43-induced neuronal cell death in vitro, whereas the expression of wt-VAPB attenuated neuronal death $^{77}$. Consistent with this, we found that P56S-VAPB leads to neuronal cell death in iPSC-derived MNs (Supplementary Fig. 1k) and a loss of nuclear localisation and cytoplasmic aggregation of fALS-associated RBPs such as FUS, TDP-43 and Matrin 3 and that of SGs in cultured cells, P56S-VAPB mice, and in ALS8 patient muscle fibres (Figs. 5 and 6). Additionally, supporting the notion that RBP homeostasis largely relies on efficient autophagy, here we confirmed that chemical inhibition of autophagy leads to extra-nuclear localisation of the majority of fALSassociated RBPs (Supplementary Fig. 3) similar to the autophagy defects mediated by P56S-VAPB.

The ALS8 patient muscle biopsy presented can be regarded as a missing piece of the puzzle. On one hand, this is valuable human tissue, because $\alpha-\mathrm{MNs}$ from P56SVAPB patients could not be examined so far because of the lack of autopsy cases. On the other hand, the considerable build-up of pTDP-43 in ALS8 muscle fibres associated with VAPB aggregates is suggestive of primary muscle involvement. This is in agreement with the emerging concept that skeletal muscle pathologies in MNDs are more than just a bystander and can actually contribute to MN degeneration (reviewed in refs. ${ }^{33,78}$ ). Such primary muscle fibre damage is also suggested by the presence of characteristic pTDP-43 aggregates in sALS muscle biopsies (Supplementary Fig. 3b), confirming earlier studies using sALS autopsy muscle ${ }^{57}$. This notion may also explain why the P56S-VAPB tg mice published so far (also included in this study), which express P56S-VAPB exclusively in the CNS, show only minor degeneration of the neuromuscular axis.

\section{Acknowledgements}

This work was supported by the Confocal Microscopy Facility, a Core Facility of the Interdisciplinary Center for Clinical Research (IZKF) Aachen within the Faculty of Medicine at RWTH Aachen University. We are grateful to the patients and their relatives for their support. We sincerely thank Stichting ALS Nederland and ALS Centre Netherlands (AE) for our ALS research support. We thank A. Knischewski and C. Krude (Institute of Neuropathology, RWTH Aachen University Hospital) for technical support and S. Gründer (Institute of Physiology, RWTH Aachen University Hospital) and his lab members for confocal microscopy. We are grateful to Prof: Anthony A. Hyman, (MPI-CGP, Munich), for G3BP-RFP stable cell line for SG analysis. This work was supported by the German Research Foundation (DFG; WE 1406/16-1 to J.W. and A.G.), the EU Joint Program Neurodegenerative Disease Research (JPND: FLY-SMALS; to J. W.), Forschungs förderung der Medizinschen Fakultät UK Aachen (START grantAZ 43/14) and Interdisciplinary Centre for Clinical Research (IZKF Aachen, N7-4), the Initiative Therapieforschung ALS e.V. and the German Society for Muscle Diseases, DGM (to J.W. and A.G.). Human IPSCs derived MNs work was supported, in part, by the NOMIS foundation to A.H. A.H. is supported by the Hermann und Lilly Schilling-Stiftung für medizinische Forschung im Stifterverband.

\section{Author details}

'Institute of Neuropathology, RWTH Aachen University Medical School, Pauwelsstr. 30, 52074 Aachen, Germany. ${ }^{2}$ EURON - European Graduate School of Neuroscience, Maastricht, The Netherlands. ${ }^{3}$ nstitute of Biomedical Engineering, Department of Cell Biology, RWTH Aachen University Medical School, Pauwelsstr. 30, 52074 Aachen, Germany. ${ }^{4}$ Department of Neurosurgery, Inselspital, Bern University Hospital, University of Bern, Bern, Switzerland. ${ }^{5}$ Institute of Biochemistry and Molecular Biology, RWTH Aachen University, 52074 Aachen, Germany. ${ }^{6}$ Confocal Microscopy Facility, Interdisciplinary Center for Clinical Research IZKF, RWTH Aachen University, 52074 Aachen, Germany. ${ }^{7}$ Amsterdam UMC, University of Amsterdam, Department of (Neuro) Pathology, Amsterdam Neuroscience, Meibergdreef 9, 1105 AZ Amsterdam, The Netherlands. ${ }^{8}$ Translational Neurodegeneration Section "Albrecht-Kossel", Department of Neurology and Center for Transdisciplinary Neurosciences Rostock (CTNR), University Medical Center Rostock, University of Rostock, 18147 Rostock, Germany. ${ }^{9}$ German Center for Neurodegenerative Diseases (DZNE) Rostock/Greifswald, 18147 Rostock, Germany. ${ }^{10}$ Department of Psychiatry and Neuropsychology, School for Mental Health and Neuroscience, Maastricht University, Maastricht, The Netherlands. "'Hämatopathologie Lübeck, Reference Centre for Lymph Node Pathology and Haematopathology, University Hospital of Schleswig-Holstein, Lübeck, Germany. ${ }^{12}$ Institute für 
Neuropathologie, Bremen-Mitte, Bremen, Germany. ${ }^{13}$ Department of Neuroscience, Erasmus MC, Rotterdam 3015 GD, The Netherlands

\section{Author contributions}

A.G. raised the hypotheses and designed the experiments. Experimental work was performed by P.T., H.G., A.D., A.Y., C.M.J. and P.D.; E.M. analysis was performed by I.K. Confocal imaging was done by A.G. Live cell imaging including FRAP analysis were performed by A.S. and S.E.; H.G. and A.H. generated and characterised and provided the Human IPSC derived MNs. Muscle biopsies from the single founder case of P56S VAPB and SALS as well as from control biopsies were examined and provided by J.W., S.N., A.C.F. and M.B. P56S transgenic mice were provided by D.J.; E.A. provided the neuropathological expertise as well as the autopsy tissue (brain, spinal cord and muscle tissue) from sALS patient. The manuscript was written by A.G. and corrected by J.W. Stylistic and conceptual corrections of the MS were performed by D.J. and H.S. All authors discussed the results and commented on the manuscript and approved the manuscript.

\section{Funding}

Open Access funding enabled and organized by Projekt DEAL.

\section{Data availability}

Available promptly upon request.

\section{Ethical approval}

The studies were approved by the Ethical Committees of the Medical Faculty, RWTH Aachen (EK 127/18), and of the Academic Medical Center, Amsterdam (W11_073).

\section{Conflict of interest}

The authors declare no competing interests.

\section{Publisher's note}

Springer Nature remains neutral with regard to jurisdictional claims in published maps and institutional affiliations.

Supplementary information The online version contains supplementary material available at https://doi.org/10.1038/s41419-021-03710-y.

Received: 30 November 2020 Revised: 12 April 2021 Accepted: 13 April 2021

Published online: 10 May 2021

\section{References}

1. Ling, S. C., Polymenidou, M. \& Cleveland, D. W. Converging mechanisms in ALS and FTD: disrupted RNA and protein homeostasis. Neuron 79, 416-438 (2013).

2. Buchan, J. R. mRNP granules. Assembly, function, and connections with disease. RNA Biol. 11, 1019-1030 (2014).

3. Nishimura, A. L. et al. A mutation in the vesicle-trafficking protein VAPB causes late-onset spinal muscular atrophy and amyotrophic lateral sclerosis. Am. J. Hum. Genet. 75, 822-831 (2004).

4. Nishimura, A. L., Al-Chalabi, A. \& Zatz, M. A common founder for amyotrophic lateral sclerosis type 8 (ALS8) in the Brazilian population. Hum. Genet. 118, 499-500 (2005).

5. Funke, A. D. et al. The p.P56S mutation in the VAPB gene is not due to a single founder: the first European case. Clin. Genet. 77, 302-303 (2010).

6. Soussan, L. et al. ERG30, a VAP-33-related protein, functions in protein transport mediated by COPI vesicles. J. Cell Biol. 146, 301-311 (1999).

7. Lev, S., Ben, H. D., Peretti, D. \& Dahan, N. The VAP protein family: from cellular functions to motor neuron disease. Trends Cell Biol. 18, 282-290 (2008).

8. Peretti, D., Dahan, N., Shimoni, E., Hirschberg, K. \& Lev, S. Coordinated lipid transfer between the endoplasmic reticulum and the Golgi complex requires the VAP proteins and is essential for Golgi-mediated transport. Mol. Biol. Cell 19, 3871-3884 (2008).
9. Amarilio, R., Ramachandran, S., Sabanay, H. \& Lev, S. Differential regulation of endoplasmic reticulum structure through VAP-Nir protein interaction. J. Biol. Chem. 280, 5934-5944 (2005).

10. Prosser, D. C., Tran, D., Gougeon, P. Y., Verly, C. \& Ngsee, J. K. FFAT rescues VAPA-mediated inhibition of ER-to-Golgi transport and VAPB-mediated ER aggregation. J. Cell Sci. 121, 3052-3061 (2008).

11. Kanekura, K., Nishimoto, I., Aiso, S. \& Matsuoka, M. Characterization of amyotrophic lateral sclerosis-linked P56S mutation of vesicle-associated membrane protein-associated protein B (VAPB/ALS8). J. Biol. Chem. 281, 30223-30233 (2006).

12. Gkogkas, C. et al. VAPB interacts with and modulates the activity of ATF6. Hum Mol. Genet. 17, 1517-1526 (2008).

13. Suzuki, H. et al. ALS-linked P56S-VAPB, an aggregated loss-of-function mutant of VAPB, predisposes motor neurons to ER stress-related death by inducing aggregation of co-expressed wild-type VAPB. J. Neurochem. 108, 973-985 (2009).

14. Zhao, Y. G. et al. The ER contact proteins VAPA/B interact with multiple autophagy proteins to modulate autophagosome biogenesis. Curr. Biol. 28, 1234-1245 (2018).

15. Kuijpers, M. et al. Amyotrophic lateral sclerosis (ALS)-associated VAPB-P56S inclusions represent an ER quality control compartment. Acta Neuropathol. Commun. 1, 24 (2013).

16. Ratnaparkhi, A., Lawless, G. M., Schweizer, F. E., Golshani, P. \& Jackson, G. R. A Drosophila model of ALS: human ALS-associated mutation in VAP33A suggests a dominant negative mechanism. PLOS ONE 3, e2334 (2008).

17. Teuling, E. et al. Motor neuron disease-associated mutant vesicle-associated membrane protein-associated protein (VAP) B recruits wild-type VAPs into endoplasmic reticulum-derived tubular aggregates. J. Neurosci. 27, 9801-9815 (2007).

18. Tran, D., Chalhoub, A., Schooley, A., Zhang, W. \& Ngsee, J. K. A mutation in VAPB that causes amyotrophic lateral sclerosis also causes a nuclear envelope defect. J. Cell Sci. 125, 2831-2836 (2012).

19. Prause, J. et al. Altered localization, abnormal modification and loss of function of Sigma receptor-1 in amyotrophic lateral sclerosis. Hum. Mol. Genet. 22, 1581-1600 (2013)

20. Gomez-Suaga, P., Paillusson, S. \& Miller, C. C. J. ER-mitochondria signaling regulates autophagy. Autophagy 13, 1250-1251 (2017).

21. Gomez-Suaga, P. et al. The ER-mitochondria tethering complex VAPB-PTPIP51 regulates autophagy. Curr. Biol. 27, 371-385 (2017).

22. Aliaga, L. et al. Amyotrophic lateral sclerosis-related VAPB P56S mutation differentially affects the function and survival of corticospinal and spinal motor neurons. Hum. Mol. Genet. 22, 4293-4305 (2013).

23. Tudor, E. L. et al. Amyotrophic lateral sclerosis mutant vesicle-associated membrane protein-associated protein-B transgenic mice develop TAR-DNAbinding protein-43 pathology. Neuroscience 167, 774-785 (2010).

24. Larroquette, F. et al. Vapb/Amyotrophic lateral sclerosis 8 knock-in mice display slowly progressive motor behavior defects accompanying ER stress and autophagic response. Hum. Mol. Genet. 24, 6515-6529 (2015).

25. Kabashi, E. et al. Investigating the contribution of VAPB/ALS8 loss of function in amyotrophic lateral sclerosis. Hum. Mol. Genet. 22, 2350-2360 (2013).

26. Ito, D., Hatano, M. \& Suzuki, N. RNA binding proteins and the pathological cascade in ALS/FTD neurodegeneration. Sci. Transl. Med. 9, eaah5436 (2017).

27. Barmada, S. J. et al. Autophagy induction enhances TDP43 turnover and survival in neuronal ALS models. Nat. Chem. Biol. 10, 677-685 (2014).

28. Brettschneider, J. et al. TDP-43 pathology and neuronal loss in amyotrophic lateral sclerosis spinal cord. Acta Neuropathol. 128, 423-437 (2014).

29. Conlon, E. G. \& Manley, J. L. RNA-binding proteins in neurodegeneration: mechanisms in aggregate. Genes Dev. 31, 1509-1528 (2017).

30. Marrone, L. et al. FUS pathology in ALS is linked to alterations in multiple ALS associated proteins and rescued by drugs stimulating autophagy. Acta Neuropathol. 138, 67-84 (2019).

31. Nachreiner, T. et al. Novel splice variants of the amyotrophic lateral sclerosisassociated gene VAPB expressed in human tissues. Biochem. Biophys. Res. Commun. 394, 703-708 (2010).

32. Jesse, C. M. et al. ALS-associated endoplasmic reticulum proteins in denervated skeletal muscle: implications for motor neuron disease pathology. Brain Pathol. 27, 781-794 (2017).

33. Goswami, A. et al. Accumulation of STIM1 is associated with the degenerative muscle fibre phenotype in ALS and other neurogenic atrophies. Neuropathol. Appl. Neurobiol. 41, 304-318 (2015) 
34. Mizushima, N., Yamamoto, A., Matsui, M., Yoshimori, T. \& Ohsumi, Y. In vivo analysis of autophagy in response to nutrient starvation using transgenic mice expressing a fluorescent autophagosome marker. Mol. Biol. Cell 15, 1101-1111 (2004).

35. Vollrath, J. T. et al. Loss of function of the ALS protein SigR1 leads to ER pathology associated with defective autophagy and lipid raft disturbances. Cell Death Dis. 12, 243 (2014).

36. Glass, $\mathrm{H}$. et al. Defective mitochondrial and lysosomal trafficking in choreaacanthocytosis is independent of Src-kinase signaling. Mol. Cell. Neurosci. 92, 137-148 (2018).

37. Naumann, M. et al. Impaired DNA damage response signaling by FUS-NLS mutations leads to neurodegeneration and FUS aggregate formation. Nat. Commun. 9, 335 (2018).

38. Mateju, D. et al. An aberrant phase transition of stress granules triggered by misfolded protein and prevented by chaperone function. EMBO J. 36, 1669-1687 (2017).

39. Poser, I. et al. BAC TransgeneOmics: a high-throughput method for exploration of protein function in mammals. Nat. Methods 5, 409-415 (2008).

40. Rapsomaniki, M. A. et al. easyFRAP: an interactive, easy-to-use tool for qualitative and quantitative analysis of FRAP data. Bioinformatics 28, 1800-1801 (2012).

41. Dubowitz, V., Serry, C. \& Oldfors, A. Muscle Biopsy A Practical Approach 4th edn. 236-240 (Saunders Ltd, 2013).

42. Dreser, A. et al. The ALS-linked E102Q mutation in Sigma receptor-1 leads to ER stress-mediated defects in protein homeostasis and dysregulation of RNAbinding proteins. Cell Death Differ. 24, 1655-1671 (2017).

43. Mitne-Neto, M. et al. Downregulation of VAPB expression in motor neurons derived from induced pluripotent stem cells of ALS8 patients. Hum. Mol. Genet. 20, 3642-3652 (2011)

44. Klionsky, D. J., Cuervo, A. M. \& Seglen, P. O. Methods for monitoring autophagy from yeast to human. Autophagy 3, 181-206 (2007).

45. Rubinsztein, D. C. et al. In search of an "autophagomometer". Autophagy 5, 585-589 (2009)

46. Klionsky, D. J. et al. Guidelines for the use and interpretation of assays for monitoring autophagy in higher eukaryotes. Autophagy 4, 151-175 (2008).

47. Klionsky, D. J. et al. Guidelines for the use and interpretation of assays for monitoring autophagy (3rd edition). Autophagy 12, 1-222 (2016).

48. Sorkin, A. et al. Recycling of epidermal growth factor-receptor complexes in A431 cells: identification of dual pathways. J. Cell Biol. 112, 55-63 (1991).

49. Kimura, S., Fujita, N., Noda, T. \& Yoshimori, T. Monitoring autophagy in mammalian cultured cells through the dynamics of LC3. Methods Enzymol. 452, 1-12 (2009).

50. Itakura, E., Kishi-Itakura, C. \& Mizushima, N. The hairpin-type tail-anchored SNARE syntaxin 17 targets to autophagosomes for fusion with endosomes/ lysosomes. Cell 151, 1256-1269 (2012).

51. Nakatogawa, H., Ichimura, Y. \& Ohsumi, Y. Atg8, a ubiquitin-like protein required for autophagosome formation, mediates membrane tethering and hemifusion. Cell 130, 165-178 (2007).

52. Xie, Z., Nair, U. \& Klionsky, D. J. Atg8 controls phagophore expansion during autophagosome formation. Mol. Biol. Cell 19, 3290-3298 (2008).

53. Mizushima, N., Yoshimori, T. \& Ohsumi, Y. The role of Atg proteins in autophagosome formation. Annu. Rev. Cell Dev. Biol. 27, 107-132 (2011).

54. Wang, X. et al. Degradation of TDP-43 and its pathogenic form by autophagy and the ubiquitin-proteasome system. Neurosci. Lett. 469, 112-116 (2010).

55. Ryu, H. H. et al. Autophagy regulates amyotrophic lateral sclerosis-linked fused in sarcoma-positive stress granules in neurons. Neurobiol. Aging 35, 2822-2831 (2014).
56. Walsh, M. J. et al. Invited review: decoding the pathophysiological mechanisms that underlie RNA dysregulation in neurodegenerative disorders: a review of the current state of the art. Neuropathol. Appl. Neurobiol. 41, 109-134 (2015).

57. Cykowski, M. D. et al. Phosphorylated TDP-43 (pTDP-43) aggregates in the axial skeletal muscle of patients with sporadic and familial amyotrophic lateral sclerosis. Acta Neuropathol. Commun. 6, 28 (2018).

58. Alberti, S., Mateju, D., Mediani, L. \& Carra, S. Granulostasis: protein quality control of RNP granules. Front. Mol. Neurosci. 10, 84 (2017).

59. Wolozin, B. Regulated protein aggregation: stress granules and neurodegeneration. Mol. Neurodegener. 7, 56 (2012).

60. Maziuk, B. F. et al. RNA binding proteins co-localize with small tau inclusions in tauopathy. Acta Neuropathol. Commun. 6, 71 (2018).

61. Nthiga, T. M. et al. CALCOCO1 acts with VAMP-associated proteins to mediate ER-phagy. EMBO J. 39, e2019103649 (2020).

62. Al-Saif, A., Al-Mohanna, F. \& Bohlega, S. A mutation in sigma-1 receptor causes juvenile amyotrophic lateral sclerosis. Ann. Neurol. 70, 913-919 (2011).

63. Senderek, J. et al. Mutations in SIL1 cause Marinesco-Sjogren syndrome, a cerebellar ataxia with cataract and myopathy. Nat. Genet. 37, 1312-1314 (2005).

64. Evgrafov, O. V. et al. Mutant small heat-shock protein 27 causes axonal Charcot-Marie-Tooth disease and distal hereditary motor neuropathy. Nat. Genet. 36, 602-606 (2004).

65. Byrne, S. et al. SIL1-related Marinesco-Sjoegren syndrome (MSS) with associated motor neuronopathy and bradykinetic movement disorder. Neuromuscul. Disord. 25, 585-588 (2015).

66. Krieger, M. et al. SIL1 mutations and clinical spectrum in patients with Marinesco-Sjogren syndrome. Brain 136, 3634-3644 (2013).

67. Capponi, S. et al. Molecular chaperones in the pathogenesis of amyotrophic lateral sclerosis: the role of HSPB1. Hum. Mutat. 37, 1202-1208 (2016).

68. Roos, A. et al. Myopathy in Marinesco-Sjogren syndrome links endoplasmic reticulum chaperone dysfunction to nuclear envelope pathology. Acta Neuropathol. 127, 761-777 (2014).

69. Herrando-Grabulosa, M., Gaja-Capdevila, N., Vela, J. M. \& Navarro, X. Sigma 1 receptor as a therapeutic target for amyotrophic lateral sclerosis. Br. J. Pharmacol. 178, 1336-1352 (2021).

70. Filezac de L'Etang, A. et al. Marinesco-Sjogren syndrome protein SIL1 regulates motor neuron subtype-selective ER stress in ALS. Nat. Neurosci. 18, 227-238 (2015).

71. Wang, C. et al. Phosphorylation of ULK1 affects autophagosome fusion and links chaperone-mediated autophagy to macroautophagy. Nat. Commun. 9 3492 (2018).

72. Gerstberger, S., Hafner, M. \& Tuschl, T. A census of human RNA-binding proteins. Nat. Rev. Genet. 15, 829-845 (2014).

73. Ramaswami, M., Taylor, J. P. \& Parker, R. Altered ribostasis: RNA-protein granules in degenerative disorders. Cell 154, 727-736 (2013).

74. Sun, Z. et al. Molecular determinants and genetic modifiers of aggregation and toxicity for the ALS disease protein FUS/TLS. PLoS Biol. 9, e1000614 (2011).

75. Buchan, J. R., Kolaitis, R. M., Taylor, J. P. \& Parker, R. Eukaryotic stress granules are cleared by autophagy and Cdc48NCP function. Cell 153, 1461-1474 (2013).

76. Monahan, Z., Shewmaker, F. \& Pandey, U. B. Stress granules at the intersection of autophagy and ALS. Brain Res. 1649, 189-200 (2016).

77. Suzuki, H. \& Matsuoka, M. Amyotrophic lateral sclerosis-linked mutant VAPB enhances TDP-43-induced motor neuronal toxicity. J. Neurochem 119, 1099-1107 (2011).

78. Boyer, J. G., Ferrier, A. \& Kothary, R. More than a bystander: the contributions of intrinsic skeletal muscle defects in motor neuron diseases. Front. Physiol. 4, 356 (2013). 